\title{
Basis Collapse for Holographic Algorithms over All Domain Sizes
}

\author{
Sitan Chen \\ Harvard University Department of Mathematics \\ Harvard John A. Paulson School of Engineering and Applied Sciences \\ Cambridge, USA \\ sitanchen@college.harvard.edu
}

\begin{abstract}
The theory of holographic algorithms introduced by Valiant represents a novel approach to achieving polynomial-time algorithms for seemingly intractable counting problems via a reduction to counting planar perfect matchings and a linear change of basis. Two fundamental parameters in holographic algorithms are the domain size and the basis size. Roughly, the domain size is the range of colors involved in the counting problem at hand (e.g. counting graph $k$ colorings is a problem over domain size $k$ ), while the basis size $\ell$ captures the dimensionality of the representation of those colors. A major open problem has been: for a given $k$, what is the smallest $\ell$ for which any holographic algorithm for a problem over domain size $k$ "collapses to" (can be simulated by) a holographic algorithm with basis size $\ell$ ? Cai and $\mathrm{Lu}$ showed in 2008 that over domain size 2, basis size 1 suffices, opening the door to an extensive line of work on the structural theory of holographic algorithms over the Boolean domain. Cai and Fu later showed for signatures of full rank that over domain sizes 3 and 4, basis sizes 1 and 2, respectively, suffice, and they conjectured that over domain size $k$ there is a collapse to basis size $\left\lfloor\log _{2} k\right\rfloor$. In this work, we resolve this conjecture in the affirmative for signatures of full rank for all $k$.
\end{abstract}

\section{Categories and Subject Descriptors}

F.2.2 [Computations on discrete structures]: Non-numerical Algorithms and Problems

\section{General Terms}

Algorithms, Theory

\section{Keywords}

Holographic algorithms, matchgates, perfect matchings, basis collapse

\footnotetext{
Permission to make digital or hard copies of all or part of this work for personal or classroom use is granted without fee provided that copies are not made or distributed for profit or commercial advantage and that copies bear this notice and the full citation on the first page. Copyrights for components of this work owned by others than ACM must be honored. Abstracting with credit is permitted. To copy otherwise, or republish, to post on servers or to redistribute to lists, requires prior specific permission and/or a fee. Request permissions from Permissions@acm.org.

Copyright is held by the owner/author(s). Publication rights licensed to ACM.

STOC'16, June 19-21, 2016, Cambridge, MA, USA

ACM. 978-1-4503-4132-5/16/06...\$15.00

http://dx.doi.org/10.1145/2897518.2897546
}

\section{INTRODUCTION}

\subsection{Matchgates and Holographic Algorithms}

In $[15,16]$, Valiant introduced the notion of matchgate computation as a method for classically simulating certain quantum gates in polynomial time and asked whether matchgates could be used to derive other polynomial-time algorithms. Given a counting problem, one might hope that its local combinatorial constraints can be encoded by graph fragments so that there exists a bijection between the number of solutions to that counting problem and the number of perfect matchings of an amalgamation $\Omega$ of those graph fragments. The celebrated Fisher-Kasteleyn-Temperley algorithm $[12,14]$ then computes the latter in polynomial time if $\Omega$ is planar.

The drawback of the above approach is that if one seeks a one-to-one reduction to counting perfect matchings in a planar graph, a matchgate-based solution might not necesssarily exist because the range of encodable local constraints is too limited. Valiant's insight in $[17,18]$ was to extend this range by looking instead for a many-to-many reduction. In his new framework, multiple strands of computation get combined in a "holographic" mixture with exponential, custom-built cancellations specified by a choice of basis vectors to produce the final answer. With this change-of-basis approach, Valiant $[17,18,19,20]$ found polynomial-time solutions to a number of counting problems, minor variants of which are known to be intractable, and noted that the only criterion for their existence was the solvability of certain finite systems of polynomial equations. As a notable example, whereas counting the number of satisfying assignments to planar, read-twice, monotone 3-CNFs is \#P-complete and even the same problem modulo 2 is NP-hard under randomized reductions, the same problem modulo 7 , known as $\#_{7} \mathrm{Pl}$-Rtw-Mon-3CNF, has a polynomial-time holographic solution [17].

Given the dearth of strong unconditional circuit lower bounds to support the prevailing belief that $\mathrm{P} \neq \mathrm{NP}$, we might question whether our current inability to produce polynomial-time solutions to NP-complete problems is sufficient to justify such a belief. After all, it would also have justified the belief that $\#_{7} \mathrm{Pl}-\mathrm{Rtw}-\mathrm{Mon}-3 \mathrm{CNF}$ is intractable. This suggests that to arrive upon the desired separation of $P$ and NP, we need a better understanding of the possibilities of polynomial-time computation. For this reason, determining the ultimate capabilities of holographic algorithms appears to be a crucial step.

To this end, one major direction has been to study the kinds of local constraints that matchgates can encode. Cai 
and his collaborators initiated a systematic study of the structural theory of holographic algorithms over the Boolean domain $[1,2,3,4,5,6,7,8,9,10]$, compiling what amounts to a catalogue of such constructions that turns the process of finding basic holographic reductions into something essentially algorithmic. For example, as a corollary to their results in [7], they noted that the modulus 7 appears in $\#_{7} \mathrm{Pl}$-Rtw-Mon-3CNF because it is a Mersenne prime and that more generally, there is a polynomial-time holographic algorithm for $\#_{2^{k}-1} \mathrm{Pl}$-Rtw-Mon- $k \mathrm{CNF}$.

\subsection{Basis Collapse Theorems}

Another direction of study concerns better understanding the power afforded by the change of basis, specifically the relationship between two important parameters of holographic algorithms: the dimension and number of basis vectors. Because holographic algorithms reduce counting problems to counting perfect matchings, the dimension of the $k$ basis vectors specifying the abovementioned "holographic mixture" must be of the form $2^{\ell}$. We say that $\ell$ is the basis size and $k$ is the domain size. The domain size should be interpreted as the range over which variables in the counting problem can take values, so for instance, problems related to counting certain $k$-colorings in a graph are problems over domain size $k$. The basis size should then be interpreted roughly as the number of bits needed to encode each of these $k$ colors.

The first holographic algorithms studied were on domain size 2, dealing with counting problems involving matchings, 2-colorings, graph bipartitions, and Boolean satisfying assignments, and used bases of size 1. The original holographic algorithm for $\#_{7} \mathrm{Pl}$-Rtw-Mon-3CNF was the first to use a basis of size 2, and at the time it may have been tempting to believe that increasing basis size adds power to the holographic approach. In [8], Cai and Lu showed to the contrary that on domain size 2 , any nontrivial holographic algorithm with basis of size $\ell \geq 2$ can be simulated by one with basis of size 1 . In other words, for all $\ell$, the class of domain size 2 problems solvable with basis size $\ell$ collapses to the class solvable with basis size 1 .

In [5], Cai and Fu further showed that holographic algorithms on domain sizes 3 and 4 using at least one full-rank signature collapse to basis sizes 1 and 2 respectively. They conjectured that for domain size $k$, we get a collapse to basis size $\left\lfloor\log _{2} k\right\rfloor$ and suggested a heuristic explanation that for domain size $k=2^{K}$, we only need $\log _{2} k$ bits to encode each of the $k$ colors.

\subsection{Our Results and Techniques}

We prove Cai and Fu's conjecture in the affirmative for all domain sizes.

ThEOREM 1.1. Any holographic algorithm on domain size $k$ using at least one matchgate with signature of rank $k$ can be simulated by a holographic algorithm with basis size $\left\lfloor\log _{2} k\right\rfloor$.

As Cai and Fu noted in [5], their "information-theoretic" explanation for the collapse theorems on domain sizes up to 4 is insufficient to explain why holographic algorithms on domain size 3 collapse to basis size 1 and not just 2 .

To prove their collapse theorem on domain size 3, Cai and $\mathrm{Fu}$ actually showed that the bases of holographic algorithms on domain size 3 which use at least one full-rank signature must be of rank 2 rather than 3 . They then invoked the main result of $\mathrm{Fu}$ and Yang [11] which reduces holographic algorithms on domain size $k$ with bases of rank 2 to ones on domain size 2 .

Our key observation is that this phenomenon occurs at a much larger scale. As a bit of informal background, the standard signature of a matchgate $G$ is a vector encoding the perfect matching properties of $G$. By indexing appropriately, we can regard the standard signature as a matrix $\Gamma$. The entries of this matrix are known [6] to satisfy a collection of quadratic polynomial identities called the Matchgate Identities (MGIs), and by using these identities together with some multilinear algebra, we prove the following:

TheOREM 1.2 (RANK Rigidity). The rank of the standard signature matrix $\Gamma$ is always a power of two.

We can then conclude that the basis of a nontrivial holographic algorithm on domain size $k$ must be of rank $2^{\ell}$, where $\ell$ is the largest integer for which $2^{\ell} \leq k$. With this step, together with a generalization of Fu and Yang's result to bases of rank $k$, we show it is enough to prove a collapse theorem for holographic algorithms on domain sizes that are powers of two. Cai and Fu [5] achieved such a collapse theorem for domain size 4 by proving that 1) any standard signature of rank 4 contains a full-rank $4 \times 4$ submatrix whose entries have indices are "close" in Hamming distance, 2) fullrank $4 \times 2^{2 n-2}$ standard signatures have right inverses that are also standard signatures.

For 1), the proof in [5] used algebraic techniques involving the matchgate identities, but these methods seem to work only for domain size 4 . We instead show that the required generalization of 1) to arbitrary domain sizes almost trivially follows from the rank rigidity theorem and the MGIs. Roughly, we prove the following:

Theorem 1.3 (Cluster Existence - Informal). If $\Gamma$ is a $2^{\ell} \times 2^{(n-1) \ell}$ matrix of rank at least $k$ realizable as the standard signature of some matchgate, then there exists a $2^{\left\lceil\log _{2} k\right\rceil} \times 2^{\left\lceil\log _{2} k\right\rceil}$ submatrix of full rank in $\Gamma$ whose column (resp. row) indices differ in at most $\left\lceil\log _{2} k\right\rceil$ bits.

For 2), the proof in [5] nonconstructively verifies that the set of all invertible $4 \times 4$ matrices satisfying the matchgate identities up to sign forms a group under multiplication. $4 \times 4$ matrices are easy to handle because there is only one nontrivial MGI in this case. Rather than generalizing this approach, we note that $\mathrm{Li}$ and Xia [13] proved a very similar but more general result under a different framework of matchgate computation known as character theory, showing that the set of all invertible $2^{K} \times 2^{K}$ matrices realizable as matchgate characters forms a group under multiplication for all $K$. It turns out their technique carries over with minor modifications into the framework of signature theory that we consider, and we use it to show the following:

Theorem 1.4 (Group Property - Informal). If $G$ is a generator matchgate with $2^{K} \times 2^{(n-1) K}$ standard signature $\underline{G}$ of rank $2^{K}$, then there exists a recognizer matchgate with $2^{K(n-1)} \times 2^{K}$ standard signature $\underline{R}$ such that $\underline{G} \cdot \underline{R}=$ $I_{2 K}$, where $I_{2^{K}}$ denotes the $2^{K} \times 2^{K}$ identity matrix.

Our general collapse theorem then follows from our generalizations of 1) and 2). This result gives a way forward for the development of the structural theory of holographic 
algorithms on higher domain sizes in the same vein as Cai et al.'s work on domain size 2. In [20], Valiant gave examples of holographic algorithms on domain size 3 , but holographic algorithms on higher domain sizes have yet to be explored. Our result shows that for domain size $k$, we can focus on

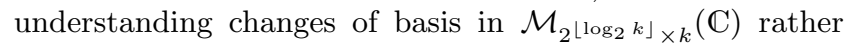
than over an infinite set of dimensions, just as the collapse theorem of Cai and $\mathrm{Lu}$ [8] showed that on the Boolean domain, they could focus on understanding changes of basis in $\mathrm{GL}_{2}(\mathbb{C})$.

\subsection{Organization}

In Section 2, we introduce the necessary notation and background, including a brief overview of holographic algorithms. In Section 3, we state the matchgate identities and some of their key consequences. In Section 4, we prove Theorems 1.2 and 1.3. In Section 5, we then prove Theorem 1.4. In Section 6, we generalize the main result of $\mathrm{Fu}$ and Yang [11] to bases of rank $k$ and reduce proving a collapse theorem on all domain sizes to proving one on domain sizes equal to powers of two. Finally, in Section 7, we prove the desired collapse theorem, Theorem 1.1, on domain sizes equal to powers of two by invoking the results from Section 4 .

\section{PRELIMINARIES}

\subsection{Background}

Denote the Hamming weight of string $\alpha$ by $\operatorname{wt}(\alpha)$, and define the parity of $\alpha$ to be the parity of $\operatorname{wt}(\alpha)$. Given $1 \leq i \leq m$, define $e_{i} \in\{0,1\}^{m}$ to be the bitstring with a single nonzero bit in position $i$. The parameter $m$ is implicit, and when this notation is used, $m$ will be clear from the context. Denote by $1^{m}$ the length- $m$ bitstring consisting solely of 1's.

We review some basic definitions and results about holographic algorithms. For a comprehensive introduction to this subject, see [18].

Definition 2.1. A matchgate $\Gamma=(G, X, Y)$ is defined by a planar embedding of a planar graph $G=(V, E, W)$, input nodes $X \subseteq V$, and output nodes $Y \subseteq V$, where $X \cap$ $Y=\emptyset$. We refer to $X \cup Y$ as the external nodes of $\Gamma$.

We say that $\Gamma$ has arity $|X|+|Y|$. In the planar embedding of $G$, the input and output nodes are arranged such that if one travels counterclockwise around the outer face of $G$, one encounters first the input nodes labeled $1,2, \ldots,|X|$ and then the output nodes $|Y|, \ldots, 2,1$.

If $\Gamma$ has exclusively output (resp. input) nodes, we say that $\Gamma$ is a generator (resp. recognizer). Otherwise, we say that $\Gamma$ is an $|X|$-input, $|Y|$-output transducer.

Definition 2.2. A basis of size $\ell$ on domain size $k$ is a $2^{\ell} \times k$ matrix $M=\left(a_{i}^{\alpha}\right)$, where rows and columns are indexed by $\alpha \in\{0,1\}^{\ell}$ and $i \in[k]$ respectively.

DeFinition 2.3. The standard signature of a matchgate $\Gamma$ of arity $n \ell$ is a vector of dimension $2^{n \ell}$ which will be denoted by $\underline{\Gamma}$, where for $\alpha_{i} \in\{0,1\}^{\ell}, \underline{\Gamma}^{\alpha_{1} \cdots \alpha_{n}}$ denotes the entry of $\underline{\Gamma}$ indexed by $\alpha_{1} \circ \cdots \circ \alpha_{n}$. If $\bar{Z}$ is the subset of the external nodes of $\Gamma$ for which $\alpha_{1} \circ \cdots \circ \alpha_{n}$ is the indicator string, then

$$
\underline{\Gamma}^{\alpha_{1} \cdots \alpha_{n}}=\operatorname{PerfMatch}(\Gamma \backslash Z) .
$$

Here, if $A=\left(A_{i j}\right)$ is the adjacency matrix of $\Gamma$, PerfMatch is the polynomial in the entries of $A$ defined by

$$
\operatorname{PerfMatch}(A)=\sum_{M} \prod_{(i, j) \in M} A_{i j},
$$

with the sum taken over the set $M$ of all perfect matchings of $\Gamma$.

The following lemma follows from the definition of standard signatures.

Lemma 2.4. Suppose $\underline{R}$ is the standard signature of a recognizer of arity $n \ell$ and $T$ the standard signature of a transducer with $s$ inputs and $\ell$ outputs. Then $\underline{R}^{\prime}=\underline{R} T^{\otimes n}$ is the standard signature of a recognizer matchgate of arity $n s$.

Definition 2.5. A column (resp. row) vector of dimension $k^{n}$ is said to be a generator (resp. recognizer) signature realizable over a basis $M$ if there exists a generator (resp. recognizer) matchgate $\Gamma$ satisfying $M^{\otimes n} G=\underline{G}$ (resp. $\underline{R} M^{\otimes n}=R$ ). We say that a collection of recognizer and generator signatures $R_{1}, \ldots, R_{a}, G_{1}, \ldots, G_{b}$ is simultaneously realizable if they are realizable over a common basis $M$.

In particular, if $M$ is square, the signature of a matchgate with respect to the standard basis is the standard signature.

Definition 2.6. A matchgrid $\Omega=(G, R, W)$ is a weighted planar graph which consists of a set of $g$ generators $G=$ $\left\{G_{1}, \ldots, G_{g}\right\}$, a set of $r$ recognizers $R=\left\{R_{1}, \ldots, R_{r}\right\}$, and a set of $w$ wires $W=\left\{W_{1}, \ldots, W_{w}\right\}$, each of which has weight 1 and connects the output node of a generator to the input node of a recognizer so that every input and output node among the matchgates in $G \cup R$ lies on exactly one wire.

Definition 2.7. Suppose $\Omega=(G, R, W)$ is a matchgrid with $g$ generators, $r$ recognizers, and $w$ wires, and let $M$ be a basis for $\Omega$. Define the Holant to be the following quantity:

$$
\operatorname{Holant}(\Omega)=\sum_{z \in[k]^{w}}\left(\prod_{i=1}^{g} G_{i}^{y_{i}} \prod_{j=1}^{r} R_{j}^{x_{j}}\right) .
$$

Here, $z=y_{1} \circ \cdots \circ y_{g}=x_{1} \circ \cdots \circ x_{r}$ such that $y_{i} \in[k]^{\left|Y_{i}\right|}$ and $x_{j} \in[k]^{\left|X_{j}\right|}$ for $Y_{i}$ the output nodes of $G_{i}$ and $X_{j}$ the input nodes of $R_{j}$, and $G_{i}$ and $R_{j}$ denote the signatures of their respective matchgates under basis $M$.

Valiant's Holant theorem states the following.

Theorem 2.8 (TheOREM 4.1, [18]). If $\Omega$ is a matchgrid over a basis $M$, then $\operatorname{Holant}(\Omega)=\operatorname{PerfMatch}(\Omega)$.

As the Fisher-Kasteleyn-Temperley algorithm $[12,14]$ can compute the number of perfect matchings of a planar graph in polynomial time, Holant $(\Omega)$ can be computed in polynomial time as long as $\Omega$ is planar.

\subsection{Matrix Form of Signatures}

It will be convenient to regard signatures not as vectors but as matrices.

Definition 2.9. For generator signature $G$, the $t$-th matrix form $G(t)(1 \leq t \leq n)$ is a $k \times k^{n-1}$ matrix where the rows are indexed by $1 \leq j_{t} \leq k$ and the columns are indexed by $j_{1} \cdots j_{t-1} j_{t+1} \cdots j_{n}$ in lexicographic order. 
Definition 2.10. For recognizer signature $R$, the $t$-th matrix form $R(t)(1 \leq t \leq n)$ is a $k^{n-1} \times k$ matrix where the rows are indexed by $j_{1} \cdots j_{t-1} j_{t+1} \cdots j_{n}$ in lexicographic order and the columns are indexed by $1 \leq j_{t} \leq k$.

We would also like to regard standard signatures as matrices; if basis $M$ is square, the following definitions are special cases of the above.

Definition 2.11. For standard signature $G$, the $t$-th matrix form $\underline{G}(t)(1 \leq t \leq n)$ is a $2^{\ell} \times 2^{(n-1) \bar{\ell}}$ matrix where the rows are indexed by $\alpha_{t}$ and the columns are indexed by $\alpha_{1} \cdots \alpha_{t-1} \alpha_{t+1} \cdots \alpha_{n}$.

Definition 2.12. For standard signature $\underline{R}$, the $t$-th matrix form $\underline{R}(t)(1 \leq t \leq n)$ is a $2^{(n-1) \ell} \times 2^{\ell}$ matrix where the rows are indexed by $\alpha_{1} \cdots \alpha_{t-1} \alpha_{t+1} \cdots \alpha_{n}$ and the columns are indexed by $\alpha_{t}$.

One can check that $\underline{G}(t)$ and $G(t)$, and $\underline{R}(t)$ and $R(t)$, are related as follows.

Lemma 2.13. Suppose $\underline{G}=M^{\otimes n} G$. It follows that $\underline{G}(t)=$ $M G(t)\left(M^{T}\right)^{\otimes(n-1)}$

LEMmA 2.14. Suppose $R=\underline{R} M^{\otimes n}$. It follows that $R(t)=$ $\left(M^{T}\right)^{\otimes(n-1)} \underline{R}(t) M$.

We will denote by $\underline{G}(t)^{\sigma}$ the row vector indexed by $\sigma$, $\underline{G}(t)_{\zeta}$ the column vector indexed by $\zeta$, and $\underline{G}(t)_{\zeta}^{\sigma}$ the entry of $\underline{G}$ in row $\sigma$ and column $\zeta$. We use analogous notation for matrices $\underline{R}, G$, and $R$. In general, if $\Gamma$ is any matrix, we will sometimes refer to the entry $\Gamma_{\zeta}^{\sigma}$ as the "entry (indexed by) $(\sigma, \zeta) . "$

In general, if $\Gamma$ is a matrix with rows indexed by $\{0,1\}^{a}$ and columns indexed by $\{0,1\}^{b}$, and $S \subset\{0,1\}^{a}$ (resp. $S \subset$ $\left.\{0,1\}^{b}\right)$, we will let $\Gamma^{S}\left(\right.$ resp. $\left.\Gamma_{S}\right)$ denote the submatrix of $\Gamma$ consisting of rows (resp. columns) indexed by $S$. Where $\Gamma$ is clear from context, we will denote the row span of $\Gamma^{S}$ (resp. column span of $\Gamma_{S}$ ) by $\operatorname{span}(S)$.

Lastly, a column/row is called odd (resp. even) if its index is odd (resp. even).

\subsection{Degenerate and Full Rank Signatures}

Definition 2.15. A signature $G$ (generator or recognizer) is degenerate iff there exist vectors $\gamma_{i}(1 \leq i \leq n)$ of dimension $k$ for which $G=\gamma_{1} \otimes \cdots \otimes \gamma_{n}$.

Lemma 2.16 (Lemma 3.1, [5]). A signature $G$ is degenerate iff $\operatorname{rank}(G(t)) \leq 1$ for $1 \leq t \leq n$.

Definition 2.17. A signature $G$ is of full rank iff there exists some $1 \leq t \leq n$ for which $\operatorname{rank}(G(t))=k$.

By Lemma 2.13, if signature $G$ is of full rank, then for the corresponding standard signature $\underline{G}$, we conclude that $\operatorname{rank}(\underline{G}(t))=k$ for some $t$. Over domain size 2, because of Lemma 2.16, all signatures not of full rank are degenerate, and holographic algorithms exclusively using such signatures are trivial because degenerate generators can by definition be decoupled into arity-1 generators. Over domain size $k \geq 3$ however, it is unknown to what extent holographic algorithms exclusively using signatures not of full rank trivialize. In [5], the collapse theorems over domain sizes 3 and 4 were proved under the assumption that at least one signature is of full rank, so we too make that assumption.

\subsection{Clusters}

One of the key results in our proof of the general collapse theorem is the existence within any matrix-form standard signature of a full-rank square submatrix whose entries have indices satisfying certain properties. In this section we make precise those properties.

Definition 2.18. A set of $2^{m}$ distinct bitstrings $Z=$ $\left\{x^{1}, \ldots, x^{2^{m}}\right\} \subset\{0,1\}^{n}$ is an $(m, n)$-cluster if there exists $s \in\{0,1\}^{n}$ and positions $p_{1}, \ldots, p_{m} \in[n]$ such that for each $i \in\left[2^{m}\right], x^{i}=s \oplus\left(\bigoplus_{j \in J} e_{p_{j}}\right)$ for some $J \subset\left\{p_{1}, \ldots, p_{m}\right\}$. We write $Z$ as $s+\left\{e_{p_{1}}, \ldots, e_{p_{m}}\right\}$. Note that for a fixed cluster, $s$ is only unique up to the bits outside of positions $p_{1}, \ldots, p_{m}$. If a cluster $Z^{\prime}$ is a subset of another cluster $Z$, we say that $Z^{\prime}$ is a subcluster of $Z$.

DeFinition 2.19. In $\Gamma$, a $2^{m} \times 2^{m}$ submatrix $\Gamma^{\prime}$ is a $m$ cluster submatrix if there exist $(m, \ell)$ - and $(m,(n-1) \ell)$ clusters $\Sigma$ and $Z$ such that $\Gamma^{\prime}=\left(\Gamma_{\zeta}^{\sigma}\right)_{\zeta \in Z}^{\sigma \in \Sigma}$ (here we omit the parameters $n$ and $\ell$ in the notation as they will be clear from context).

\section{MATCHGATE IDENTITIES AND CON- SEQUENCES}

\subsection{Parity Condition and Matchgate Identities}

The most obvious property that standard signatures satisfy is the parity condition: because a graph with an odd number of vertices has no perfect matchings, the indices of the nonzero entries in $\underline{G}$ have the same parity. It trivially follows that in $\underline{G}(t)$, columns $\underline{G}(t)_{\zeta}$ and $\underline{G}(t)_{\eta}$, if both nonzero, are linearly independent if $\zeta$ and $\eta$ are of opposite parities. The same holds for the rows of $\underline{G}(t)$.

In [6] it is shown algebraically that the parity condition in fact follows from the so-called matchgate identities which we present below, quadratic relations which together form a necessary and sufficient condition for a vector to be the standard signature of some matchgate.

As in Cai and Fu's proof of the collapse theorem for domain size 4, we will make heavy use of the matchgate identities stated below. Wherever we invoke them, they will be for generator matchgates, so we focus on this case.

TheOREM 3.1 (THEOREM 2.1, [6]). A $2^{\ell} \times 2^{(n-1) \ell} m a-$ trix $\Gamma$ is the $t$-th matrix form of the standard signature of some generator matchgate iff for all $\zeta, \eta \in\{0,1\}^{(n-1) \ell}$ and $\sigma, \tau \in\{0,1\}^{\ell}$, the following matchgate identity holds. Let $\zeta \oplus \eta=e_{q_{1}} \oplus \cdots \oplus e_{q_{d^{\prime}}}$ and $\sigma \oplus \tau=e_{p_{1}} \oplus \cdots \oplus e_{p_{d}}$, where $q_{1}<\cdots<q_{d^{\prime}}$ and $p_{1}<\cdots<p_{d}$. Then

$$
\begin{aligned}
\sum_{i=1}^{d}(-1)^{i+1} \Gamma_{\zeta}^{\left(\sigma \oplus e_{p_{1}} \oplus e_{p_{i}}\right)} \Gamma_{\eta}^{\left(\tau \oplus e_{p_{1}} \oplus e_{p_{i}}\right)}= \\
\sum_{j=1}^{d^{\prime}} \pm \Gamma_{\left(\zeta \oplus e_{q_{j}}\right)}^{\left(\sigma \oplus e_{p_{1}}\right)} \Gamma_{\left(\eta \oplus e_{q_{j}}\right)}^{\left(\tau \oplus e_{p_{1}}\right)}
\end{aligned}
$$

Here the \pm signs depend on both $j$ and, if $q_{j}$ is after the $t$-th 
block, the parity of $d$. If $d$ is even,

$$
\begin{aligned}
\sum_{i=1}^{d}(-1)^{i+1} \Gamma_{\zeta}^{\left(\sigma \oplus e_{p_{1}} \oplus e_{p_{i}}\right)} \Gamma_{\eta}^{\left(\tau \oplus e_{p_{1}} \oplus e_{p_{i}}\right)}= \\
\epsilon_{\zeta, \eta} \sum_{j=1}^{d^{\prime}}(-1)^{j+1} \Gamma_{\left(\zeta \oplus e_{q_{j}}\right)}^{\left(\sigma \oplus e_{p_{1}}\right)} \Gamma_{\left(\eta \oplus e_{q_{j}}\right)}^{\left(\tau \oplus e_{p_{1}}\right)},
\end{aligned}
$$

where $\epsilon_{\zeta, \eta} \in\{ \pm 1\}$ is positive (resp. negative) if the number of $q_{j}$ preceding the $t$-th block is odd (resp. even).

REMARK 3.2. If $d+d^{\prime}$ is odd, (1) is trivial by the parity condition.

We will be making extensive use of the matchgate identities in this paper, but we will typically not care about the $\epsilon_{\zeta, \eta}$ sign on the right-hand side of (2). For this reason, it will be convenient to make the following definition.

Definition 3.3. A $2^{\ell} \times 2^{m}$ matrix $M$ is a pseudo-signature if for all $\sigma, \tau$ for which wt $\sigma \oplus \tau)$ is even, its entries satisfy the corresponding identity (2) up to a factor of \pm 1 on the right-hand side.

Standard signatures and cluster submatrices are all examples of pseudo-signatures.

OBSERVATION 1. If $M$ is a pseudo-signature, then $M^{T}$ is a pseudo-signature.

\subsection{Matchgate Identities and Determinants}

We now derive from the matchgate identities some basic linear algebraic properties of the columns of pseudosignatures. By Observation 1, these also apply for the rows.

Firstly, we have the following immediate consequence of Theorem 3.1. We specifically consider the case where wt $(\zeta \oplus$ $\eta)$ is even and, by Remark 3.2, $\operatorname{wt}(\sigma \oplus \tau)$ is even. So write $\zeta \oplus \eta=e_{q_{1}} \oplus \cdots \oplus e_{q_{2 d^{\prime}}}$ and $\sigma \oplus \tau=e_{p_{1}} \oplus \cdots \oplus e_{p_{2 d}}$.

Reverse the roles of $\zeta$ and $\eta$ in (2). Subtract the resulting equation from (2) to find

$$
\begin{aligned}
& \sum_{i=1}^{2 d}(-1)^{i+1}\left|\begin{array}{ll}
\Gamma_{\zeta}^{\left(\sigma \oplus e_{p_{1}} \oplus e_{p_{i}}\right)} & \Gamma_{\eta}^{\left(\sigma \oplus e_{p_{1}} \oplus e_{p_{i}}\right)} \\
\Gamma_{\zeta}^{\left(\tau \oplus e_{p_{1}} \oplus e_{p_{i}}\right)} & \Gamma_{\eta}^{\left(\tau \oplus e_{p_{1}} \oplus e_{p_{i}}\right)}
\end{array}\right|= \\
& \epsilon_{\zeta, \eta} \sum_{j=1}^{2 d^{\prime}}(-1)^{j+1}\left|\begin{array}{ll}
\Gamma_{\left(\zeta \oplus e_{q_{j}}\right)}^{\left(\sigma \oplus e_{p_{1}}\right)} & \Gamma_{\left(\eta \oplus e_{q_{j}}\right)}^{\left(\sigma \oplus e_{p_{1}}\right)} \\
\Gamma_{\left(\zeta \oplus e_{q_{j}}\right)}^{\left(\tau \oplus e_{p_{1}}\right)} & \Gamma_{\left(\eta \oplus e_{q_{j}}\right)}^{\left(\tau \oplus e_{p_{1}}\right)}
\end{array}\right|
\end{aligned}
$$

Example 1. Suppose $d=d^{\prime}=1$. Then (3) becomes

$$
2\left|\begin{array}{cc}
\Gamma_{\zeta}^{\sigma} & \Gamma_{\eta}^{\sigma} \\
\Gamma_{\zeta}^{\tau} & \Gamma_{\eta}^{\tau}
\end{array}\right|=2 \epsilon_{\zeta, \eta}\left|\begin{array}{cc}
\Gamma_{\zeta \oplus e_{q_{1}}}^{\sigma \oplus e_{p_{1}}} & \Gamma_{\zeta \oplus e_{p_{1}}}^{\sigma \oplus e_{q_{2}}} \\
\Gamma_{\zeta \oplus e_{q_{1}}}^{\sigma \oplus e_{q_{2}}} & \Gamma_{\zeta \oplus e_{q_{2}}}^{\sigma \oplus e_{2}}
\end{array}\right|
$$

so in particular, the matrix on the left is singular iff the latter is.

More generally, only suppose that $d=1$. Then (3) becomes

$$
2\left|\begin{array}{cc}
\Gamma_{\zeta}^{\sigma} & \Gamma_{\eta}^{\sigma} \\
\Gamma_{\zeta}^{\tau} & \Gamma_{\eta}^{\tau}
\end{array}\right|=\epsilon_{\zeta, \eta} \sum_{j=1}^{2 d^{\prime}}(-1)^{j+1}\left|\begin{array}{ll}
\Gamma_{\left(\zeta \oplus e_{q_{j}}\right)}^{\left(\sigma \oplus e_{p_{1}}\right)} & \Gamma_{\left(\eta \oplus e_{q_{j}}\right)}^{\left(\sigma \oplus e_{p_{1}}\right)} \\
\Gamma_{\left(\zeta \oplus e_{q_{j}}\right)}^{\left(\sigma \oplus e_{p_{2}}\right)} & \Gamma_{\left(\eta \oplus e_{q_{j}}\right)}^{\left(\sigma \oplus e_{p_{2}}\right)}
\end{array}\right|
$$

so in particular, the matrix on the left-hand side is singular if all $2 d^{\prime}$ matrices on the right-hand side are singular.
In other words, if $\ell=2$ so that $\Gamma$ only has four rows, columns $\zeta$ and $\eta$ as defined above are linearly dependent if all pairs of neighboring columns are linearly dependent. We shall see in the next section (Corollary 3.5) that this is true even when $\Gamma$ has an arbitrary number of rows.

\subsection{Wedge Products of Columns}

Motivated by Example 1, we'd like to study the set of all $2 \times 2$ determinants $\left|\begin{array}{cc}\Gamma_{\zeta}^{\sigma} & \Gamma_{\eta}^{\sigma} \\ \Gamma_{\zeta}^{\tau} & \Gamma_{\eta}^{\tau}\end{array}\right|$ given two column vectors $\Gamma_{\zeta}$ and $\Gamma_{\eta}$ of the same parity. These are merely the coefficients of the wedge product $\Gamma_{\zeta} \wedge \Gamma_{\eta}$ under the standard basis $\left\{v_{\sigma} \wedge v_{\tau}\right\}_{\sigma, \tau \in\{0,1\}^{2^{\ell}}, \sigma<\tau}$ (where the relation $\sigma<\tau$ denotes lexicographic ordering) of $\bigwedge^{2} \mathbb{C}^{2^{\ell}}$, the second exterior power of $\mathbb{C}^{2^{\ell}}$. The matchgate identities imply the following consequence about the relationships among the wedge products $\Gamma_{\zeta} \wedge \Gamma_{\eta}$ as $\zeta$ and $\eta$ vary.

LEMmA 3.4. If $\zeta_{1}, \eta_{1}, \zeta_{2}, \eta_{2}, \ldots, \zeta_{m}, \eta_{m}$ are even indices for which

$$
\sum_{\nu=1}^{m} a_{\nu}\left(\Gamma_{\zeta_{\nu}} \wedge \Gamma_{\eta_{\nu}}\right)=0
$$

for some $a_{1}, \ldots, a_{m} \in \mathbb{C}$, then

$$
\sum_{\nu=1}^{m} \epsilon_{\zeta_{\nu}, \eta_{\nu}} a_{\nu}\left(\sum_{i=1}^{2 d_{\nu}}(-1)^{j+1} \Gamma_{\zeta_{\nu} \oplus e_{p_{i}^{\nu}}} \wedge \Gamma_{\eta_{\nu} \oplus e_{p_{i}^{\nu}}}\right)=0,
$$

where for each $\nu, \operatorname{wt}\left(\zeta_{\nu} \oplus \eta_{\nu}\right)=2 d_{\nu}$ and $\zeta_{\nu} \oplus \eta_{\nu}=e_{p_{1}^{\nu}} \oplus$ $\cdots \oplus e_{p_{2 d_{\nu}}^{\nu}}$.

Proof. For convenience, we will denote $\epsilon_{\zeta_{\nu}, \eta_{\nu}}$ by $\epsilon_{\nu}$. First, we rewrite the left-hand side of (6) in terms of coordinates as

$$
\sum_{\nu=1}^{m} \epsilon_{\nu} a_{\nu}\left(\sum_{i=1}^{2 d_{\nu}}(-1)^{i+1} \sum_{\sigma<\tau}\left|\begin{array}{ll}
\Gamma_{\zeta_{\nu} \oplus e_{p_{i}^{\nu}}}^{\sigma} & \Gamma_{\eta_{\nu} \oplus e_{p_{i}^{\nu}}}^{\sigma} \\
\Gamma_{\zeta_{\nu} \oplus e_{p_{i}^{\nu}}}^{\tau} & \Gamma_{\eta_{\nu} \oplus e_{p_{i}^{\nu}}}^{\tau}
\end{array}\right|\left(v_{\sigma} \wedge v_{\tau}\right)\right)
$$

where $\sigma<\tau$ denotes lexicographical ordering. Note that the determinants that appear in the left-hand side of (7) are zero when $\sigma$ and $\tau$ are of opposite parity. Moreover, depending on the parity of the signature $\Gamma$, either all such determinants are also zero for $\sigma$ and $\tau$ even, or they are all zero for $\sigma$ and $\tau$ odd.

Rearranging the order of summations, we can express (7) as

$\sum_{\sigma<\tau}\left(v_{\sigma} \wedge v_{\tau}\right) \cdot\left(\sum_{\nu=1}^{m} \epsilon_{\nu} a_{\nu} \sum_{i=1}^{2 d_{\nu}}(-1)^{i+1}\left|\begin{array}{cc}\Gamma_{\zeta_{\nu} \oplus e_{p_{i}^{\nu}}}^{\sigma} & \Gamma_{\eta_{\nu} \oplus e_{p_{i}^{\nu}}}^{\sigma} \\ \Gamma_{\zeta_{\nu} \oplus e_{p_{i}^{\nu}}}^{\tau} & \Gamma_{\eta_{\nu} \oplus e_{p_{i}^{\nu}}}^{\tau}\end{array}\right|\right)$

For a fixed pair $\sigma<\tau$, let $\operatorname{wt}(\sigma \oplus \tau)=2 d^{\prime}$ and $\sigma \oplus \tau=$ $e_{q_{1}} \oplus \cdots \oplus e_{q_{2 d^{\prime}}}$. If we apply (3) and rearrange the order of summation once more, the coefficient of $v_{\sigma} \wedge v_{\tau}$ above becomes

$$
\begin{gathered}
\sum_{\nu=1}^{m} a_{\nu} \cdot \sum_{j=1}^{2 d^{\prime}}(-1)^{j+1}\left|\begin{array}{ll}
\Gamma_{\zeta_{\nu}}^{\sigma \oplus e_{j}} & \Gamma_{\eta_{\nu}}^{\sigma \oplus e_{q_{j}}} \\
\Gamma_{\zeta_{\nu}}^{\tau \oplus e_{q_{j}}} & \Gamma_{\eta_{\nu}}^{\tau \oplus e_{q_{j}}}
\end{array}\right|= \\
\sum_{j=1}^{2 d^{\prime}}(-1)^{j+1} \sum_{\nu=1}^{m} a_{\nu}\left|\begin{array}{cc}
\Gamma_{\zeta_{\nu}}^{\sigma \oplus e_{q_{j}}} & \Gamma_{\eta_{\nu}}^{\sigma \oplus e_{q_{j}}} \\
\Gamma_{\zeta_{\nu}}^{\tau \oplus e_{q_{j}}} & \Gamma_{\eta_{\nu}}^{\tau \oplus e_{q_{j}}}
\end{array}\right|
\end{gathered}
$$

But note that if we expand (5) in terms of coordinates, the 
term

$$
a_{\nu}\left|\begin{array}{ll}
\Gamma_{\zeta_{\nu}}^{\sigma \oplus e_{q_{j}}} & \Gamma_{\eta_{\nu}}^{\sigma \oplus e_{q_{j}}} \\
\Gamma_{\zeta_{\nu}}^{\tau \oplus e_{q_{j}}} & \Gamma_{\eta_{\nu}}^{\tau \oplus e_{q_{j}}}
\end{array}\right|
$$

is precisely the coefficient of $v_{\sigma \oplus e_{q_{j}}} \wedge v_{\tau \oplus e_{q_{j}}}$ in the expansion of (5) in terms of coordinates and hence zero by assumption, so (8) holds as desired.

COROLlary 3.5. Let $\zeta, \eta \in\{0,1\}^{(n-1) \ell}$ be such that $\zeta \oplus$ $\eta=\bigoplus_{j=1}^{2 d} e_{p_{i}}$. If column $\Gamma_{\left(\zeta \oplus e_{p_{i}}\right)}$ is linearly dependent with column $\Gamma_{\left(\eta \oplus e_{p_{i}}\right)}$ for $1 \leq i \leq 2 d$, then column $\Gamma_{\zeta}$ is linearly dependent with column $\Gamma_{\eta}$.

Corollary 3.6. Let $\zeta, \eta \in\{0,1\}^{(n-1) \ell}$ be such that $\zeta \oplus$ $\eta=\bigoplus_{i=1}^{2 d} e_{p_{i}}$. If there exists $i \in\left[2 d^{\prime}\right]$ such that column $\Gamma_{\zeta \oplus e_{p_{i}}}$ is linearly dependent with column $\Gamma_{\eta \oplus e_{p_{i}}}$ for $i=$ $1, \ldots, \hat{j}, \ldots, 2 d^{\prime}$, where $\hat{j}$ denotes omission of index $j$, and if $\Gamma_{\zeta}$ is also linearly dependent with column $\Gamma_{\eta}$, then $\Gamma_{\zeta \oplus e_{p_{j}}}$ and $\Gamma_{\eta \oplus e_{p_{j}}}$ are linearly dependent.

Lemma 3.4 says that any linear relation among wedges of even columns yields a linear relation among wedges of odd columns, and vice versa.

Lastly, we need the following elementary result in multilinear algebra.

LEммA 3.7. If $v_{1}, \ldots, v_{n}$ are linearly independent in vector space $V$, then the set of all $v_{i} \wedge v_{j}$ for $i<j$ are linearly independent in $\bigwedge^{2} V$.

Combining this with Lemma 3.4 yields the following key ingredient to the analysis in Section 4.

Lemma 3.8. Suppose $\zeta_{0}, \eta \in\{0,1\}^{K}$ such that $\zeta_{0} \neq \eta$, and the indices in $T=\left\{\zeta_{1}, \ldots, \zeta_{m}\right\} \subset\{0,1\}^{K}$ are distinct and have the same parity. Suppose further that $\zeta_{0} \neq \zeta_{1}, \ldots, \zeta_{m}$. Let $\zeta_{i} \oplus \eta=e_{p_{1}^{i}} \oplus \cdots \oplus e_{p_{d_{i}}}$ for $0 \leq i \leq m$, where $d_{i}:=$ $\operatorname{wt}\left(\zeta_{i} \oplus \eta\right)$. Define

$$
S:=\bigcup_{0 \leq i \leq m, 1 \leq j \leq d_{i}}\left\{\eta \oplus e_{p_{j}^{i}}, \zeta_{i} \oplus e_{p_{j}^{i}}\right\} \subset\{0,1\}^{K}
$$

not in the sense of multisets, that is, we throw out duplicates so that the strings in $S$ are all distinct.

Suppose the columns indexed by $S$ are linearly independent. Then

$$
\Gamma_{\zeta_{0}} \notin \operatorname{span}\left(\Gamma_{\zeta_{1}}, \ldots, \Gamma_{\zeta_{m}}\right)
$$

If $\operatorname{wt}\left(\eta \oplus \zeta_{0}\right) \geq 4$ and $j^{*} \in\left[d_{0}\right]$, then (10) holds even if only the columns indexed by $S^{\prime}:=S \backslash\left\{\zeta_{0} \oplus e_{p_{j^{*}}^{0}}\right\}$ are linearly independent.

Proof. We first prove the claim without the assumption that $\operatorname{wt}\left(\eta \oplus \zeta_{0}\right) \geq 4$. Suppose to the contrary that $\Gamma_{\zeta_{0}}=$ $\sum_{i=1}^{m} a_{i} \Gamma_{\zeta_{i}}$ so that $\Gamma_{\zeta_{0}} \wedge \Gamma_{\eta}-\sum_{i=1}^{m} a_{i} \Gamma_{\zeta_{i}} \wedge \Gamma_{\eta}=0$. By Lemma 3.4, this linear relation implies the following linear relation among wedges of columns of the other parity:

$$
\begin{aligned}
& \epsilon_{\zeta_{0}, \eta}\left(\sum_{j=1}^{d_{0}}(-1)^{j+1} \Gamma_{\zeta_{0} \oplus e_{p_{j}^{0}}} \wedge \Gamma_{\eta \oplus e_{p_{j}^{0}}}\right)- \\
& \sum_{i=1}^{m} \epsilon_{\zeta_{i}, \eta} a_{i}\left(\sum_{j=1}^{d_{i}}(-1)^{j+1} \Gamma_{\zeta_{i} \oplus e_{p_{j}^{i}}} \wedge \Gamma_{\eta \oplus e_{p_{j}^{i}}}\right)=0
\end{aligned}
$$

We claim this is a nontrivial linear relation contradicting the linear independence of the columns indexed by $S$. For each of the $m+1$ sums indexed by $1 \leq j \leq d_{i}$ appearing in (11), if $d_{i}=2$, rewrite $\sum_{j=1}^{d_{i}}(-1)^{j+1} \Gamma_{\zeta_{i} \oplus e_{p_{j}^{i}}} \wedge \Gamma_{\eta \oplus e_{p_{j}^{i}}}$ as $2 \Gamma_{\zeta_{i} \oplus e_{p_{1}^{i}}} \wedge \Gamma_{\eta \oplus e_{p_{1}^{i}}}$.

After this consolidation, note that the wedge products in (11) are now all distinct. Certainly for any $j, j^{\prime} \in\left[d_{i}\right]$ where $d_{i} \geq 4, \Gamma_{\zeta_{i} \oplus e_{p_{j}^{i}}} \wedge \Gamma_{\eta \oplus e_{p_{j}^{i}}}$ and $\Gamma_{\zeta_{i} \oplus e_{p_{j^{\prime}}}} \wedge \Gamma_{\eta \oplus e_{p_{j^{\prime}}}}$ are linearly independent. For $i, i^{\prime}$ such that $d_{i}=2$ and $d_{i^{\prime}}>2$, $2 \Gamma_{\zeta_{i} \oplus e_{p_{1}^{i}}} \wedge \Gamma_{\eta \oplus e_{p_{1}^{i}}}$ and any $\Gamma_{\zeta_{i^{\prime}} \oplus e_{p_{j}^{i^{\prime}}}} \wedge \Gamma_{\eta \oplus e_{p_{j}^{i^{\prime}}} \text { are linearly }}$ independent as $\zeta_{i} \oplus \eta \neq \zeta_{i^{\prime}} \oplus \eta$, contradicting the assumption that $\left\{\zeta_{1}, \ldots, \zeta_{m}\right\}$ are distinct. Similarly, for $i, i^{\prime}$ such that $d_{i}>2$ and $d_{i^{\prime}}>2$, any $\Gamma_{\zeta_{i} \oplus e_{p_{j}^{i}}} \wedge \Gamma_{\eta \oplus e_{p_{j}^{i}}}$ and any $\Gamma_{\zeta_{i^{\prime}} \oplus e_{p_{j^{\prime}}^{i^{\prime}}}} \wedge$ $\Gamma_{\eta \oplus e_{p_{j^{\prime}}^{i^{\prime}}}}$ are linearly independent as $\zeta_{i} \oplus \eta \neq \zeta_{i^{\prime}} \oplus \eta$.

We conclude that (11), after consolidating sums for which $d_{i}=2$, consists of a nonzero number of linearly independent wedge products of columns indexed by $S$, so (11) is indeed a nontrivial linear relation among the wedge products $\Gamma_{s} \wedge \Gamma_{s^{\prime}}$ for $s, s^{\prime} \in S$. But all columns indexed by $S$ are linearly independent by assumption, so this linear relation contradicts Lemma 3.7 and the linear independence of columns indexed by $S$.

For the second part of Lemma 3.8, we claim that (11) is still a nontrivial relation. Pick any $k \neq j^{*}$ inside $\left[d_{0}\right]$. Because $d_{0} \geq 4$,

$$
\zeta_{0} \oplus e_{p_{k}^{0}}, \eta \oplus e_{p_{k}^{0}} \neq \eta \oplus e_{p_{j^{*}}^{0}} .
$$

We have already taken care of the case where $\Gamma_{\zeta_{0}+e_{p_{j^{*}}}} \notin$ $\operatorname{span}\left(S^{\prime}\right)$ above, so suppose instead that

$$
\Gamma_{\zeta_{0}+e_{p_{j^{*}}^{0}}}=\sum_{s \in S^{\prime}} b_{s} \Gamma_{s}
$$

If we consolidate sums for which $d_{i}=2$ in (11) as above and substitute (13) into the resulting equation, the wedge products that (11) now contains also include ones of the form $\Gamma_{s} \wedge \Gamma_{\eta+e_{p_{j^{*}}}}$, which cannot be linearly dependent with $\Gamma_{\zeta_{0} \oplus e_{p_{k}^{0}}} \wedge \Gamma_{\eta \oplus e_{p_{k}^{0}}}$ by (12).

Every other wedge product in (11) is of the form $\Gamma_{\zeta_{i} \oplus e_{p_{j}^{i}}} \wedge$ $\Gamma_{\eta \oplus e_{p_{j}^{i}}}$ and also cannot be linearly dependent with $\pm \Gamma_{\zeta_{0} \oplus e_{p_{k}^{0}}} \wedge$ $\Gamma_{\eta \oplus e_{p_{k}^{0}}}$ or else, as before, we'd find that $\zeta_{i} \oplus \eta=\zeta_{k} \oplus \eta$, contradicting the assumption that $\left\{\zeta_{1}, \ldots, \zeta_{m}\right\}$ are distinct.

It follows that if we rewrite the left-hand side of (11) in the form $\sum_{s, s^{\prime} \in S^{\prime}} b_{s, s^{\prime}} \Gamma_{s} \wedge \Gamma_{s^{\prime}}$ (uniquely because by Lemma 3.7 the $\Gamma_{s} \wedge \Gamma_{s^{\prime}}$ are linearly independent), $b_{\zeta_{0} \oplus e_{p_{k}^{0}}, \eta \oplus e_{p_{k}^{0}}} \neq 0$. So (11) is still a nontrivial linear relation, contradicting Lemma 3.7 and the linear independence of columns indexed by $S$.

\section{RIGIDITY AND CLUSTER EXISTENCE}

In this section, we will prove the rigidity theorem and the cluster existence theorem, informally stated as Theorems 1.2 and 1.3. Now that we have introduced the appropriate terminology, we first state both precisely.

TheOrem 4.1 (RANK Rigidity - Formal). If $\Gamma$ is a $2^{\ell} \times 2^{m}$ pseudo-signature, then $\operatorname{rank}(\Gamma)$ is a power of 2 . 
Equivalently, for all $\kappa \geq 1$,

$$
\operatorname{rank}(\Gamma) \geq 2^{\kappa}+1 \Rightarrow \operatorname{rank}(\Gamma) \geq 2^{\kappa+1}
$$

Theorem 4.2 (Cluster Existence - Formal). If $\Gamma=$ $\underline{G}(t)$ for some matchgate $G$ of arity $n \ell$, and $\operatorname{rank}(\Gamma) \geq k$, then there is a $\left\lceil\log _{2} k\right\rceil$-cluster submatrix of full rank.

To prove Theorem 4.2, we claim it is enough to show the following:

THEOREM 4.3. If $\Gamma$ is a $2^{\ell} \times 2^{m}$ pseudo-signature such that $\operatorname{rank}(\Gamma) \geq k$, then there exists a $\left(\left\lceil\log _{2} k\right\rceil, m\right)$-cluster $Z$ for which $\Gamma_{Z}$ is of full rank.

Equivalently, for all $\kappa \geq 1$,

$$
\begin{aligned}
& \operatorname{rank}(\Gamma) \geq 2^{\kappa}+1 \Rightarrow \\
& \exists(\kappa+1, m) \text {-cluster } Z \text { such that } \operatorname{rank}\left(\Gamma_{Z}\right)=2^{\kappa+1}
\end{aligned}
$$

Proof of Theorem 4.2. Apply Theorem 4.3 to $\Gamma$ to obtain $\Gamma_{Z}$. By Observation $1, \Gamma_{Z}^{T}$ is also a pseudo-signature. Apply Theorem 4.3 to $\Gamma^{T}$ to get the desired cluster submatrix.

Note that for fixed $\kappa,(15)$ implies (14). We will jointly prove Theorem 4.1 and Theorem 4.3 by induction on $k$. Cai and $\mathrm{Fu}$ have already shown both for $k \leq 4$; we take these results as our base case. We complete the following two inductive steps.

INDUCTIVE STEP 1. If implication (15) holds for $1 \leq \kappa \leq$ $K-1$, then implication (14) holds for $1 \leq \kappa \leq K$.

INDUCTIVE STEP 2. If implication (14) holds for $1 \leq \kappa \leq$ $K$ and implication (15) holds for $\kappa \leq K-1$, then implication (15) also holds for $\kappa=K$.

Note that once we have proven the rigidity and cluster existence theorems, we additionally obtain the following.

COROLlary 4.4. If $G$ is a full-rank matchgate signature on domain size $k$, it is only realizable over bases $M$ of rank at most $2^{\left\lfloor\log _{2} k\right\rfloor}$.

Proof. If $k$ is a power of two, the claim is trivial. If not, assume $G$ is a generator (standard signatures of recognizer are also pseudo-signatures, so the argument in that case is analogous). If $\operatorname{rank}(G(t))=k$ and $\operatorname{rank}(M) \geq 2^{\left\lfloor\log _{2} k\right\rfloor}+1$, then by Lemma 2.14, $2^{\left\lfloor\log _{2} k\right\rfloor}+1 \leq \operatorname{rank}(\underline{G}(t)) \leq k$. But Theorem 4.1 would then imply $\operatorname{rank}(\underline{G}(t)) \geq 2^{\left\lfloor\log _{2} k\right\rfloor+1}>k$, a contradiction.

\subsection{Rank Rigidity Theorem}

In this subsection, we complete the former inductive step, and in the next, we complete the latter.

Before we prove Inductive Step 1 in its entirety, we take care of the case where $m=K+1$. While this might appear to be the simplest case because $m$ is minimal, it will turn out that cases where $m$ is greater will reduce to this case. For this reason, the wedge product machinery introduced in Section 3 is used exclusively in the proof of this case.

Once we take care of this case, we will essentially show that if standard signature $\Gamma$ is any wider, i.e. if $m>K+1$, then if $Z$ is a cluster of size greater than $2^{K}$ indexing columns of rank at least $2^{K}+1$, we can always find a proper subcluster also indexing columns of rank at least $2^{K}+1$, or else the matchgate identities would erroneously imply that certain columns which are known to be linearly independent are linearly dependent.

We begin with the case of $m=K+1$.

THEOREM 4.5. If $\Gamma$ is a $2^{\ell} \times 2^{K+1}$ pseudo-signature such that $\operatorname{rank}(\Gamma) \geq 2^{K}+1$, then $\operatorname{rank}(\Gamma)=2^{K+1}$.

Proof. Because we assume implication (15) holds for $\kappa=K-1, \Gamma$ contains a $(K, m)$-cluster of linearly independent columns $Z=s \oplus\left\{e_{1}, \ldots, \hat{e_{j}}, \ldots, e_{K+1}\right\}$; denote the even indices of $Z$ by $Z_{0}$ and the odd ones by $Z_{1}$. Because $\operatorname{rank}(\Gamma)>2^{K}$, there exists $t \notin Z$ for which $\Gamma_{t} \notin \operatorname{span}(Z)$. Denote the parity of $t$ by $b \in\{0,1\}$, and denote by $\bar{b}$ the opposite parity.

Select any $t^{\prime}=t \oplus e_{i^{*}}$ for $i^{*} \neq j$ and apply Lemma 3.8 to $\zeta_{0}=t^{\prime}, \eta=t \oplus e_{j}, T=Z_{\bar{b}}$ to conclude that $\Gamma_{t^{\prime}} \notin \operatorname{span}\left(Z_{\bar{b}}\right)$.

Let $S_{d_{1}, d_{2}}$ denote the set of column indices $\zeta \notin Z$ for which $\operatorname{wt}(\zeta \oplus t)=d_{1}$ and $\operatorname{wt}\left(\zeta \oplus t^{\prime}\right)=d_{2}$. Note that because $\operatorname{wt}\left(t \oplus t^{\prime}\right)=1, S_{d_{1}, d_{2}}$ is empty if $\left|d_{1}-d_{2}\right| \neq 1$.

We will show by induction on $D$ that the columns indexed by $\left(\cup_{d=0}^{D} S_{d, d+1} \cup S_{d+1, d}\right) \cup Z$ are linearly independent for all $0 \leq D \leq K$. The definition of $t$ and the argument above for $t^{\prime}$ give the base case of $D=0$.

For the inductive step, for each $d$ let $d_{0}$ and $d_{1}$, respectively denote the even and odd value in $\{d, d+1\}$. As columns indexed by $S_{d_{0}, d_{1}}$ and columns indexed by $S_{d_{1}, d_{0}}$ have opposite parity, it suffices to show that the columns indexed by $T_{D}^{0}:=\left(\bigcup_{d=0}^{D} S_{d_{0}, d_{1}}\right) \cup Z_{b}$ are linearly independent, and that the columns indexed by $T_{D}^{1}:=\left(\cup_{d=0}^{D} S_{d_{1}, d_{0}}\right) \cup Z_{\bar{b}}$ are linearly independent.

Within this inductive step, we will further induct on the elements within $S_{D_{0}, D_{1}}$ and $S_{D_{1}, D_{0}}$. Specifically, suppose we have already proven that for some subset $S_{D_{0}, D_{1}}^{\prime} \subset S_{D_{0}, D_{1}}$, all columns indexed by $T_{D-1}^{0} \cup S_{D_{0}, D_{1}}^{\prime}$ are linearly independent, and that for $S_{D_{1}, D_{0}}^{\prime}:=\left\{u \oplus e_{i^{*}}: u \in S_{D_{0}, D_{1}}^{\prime}\right\} \subset$ $S_{D_{1}, D_{0}}$, all columns indexed by $T_{D-1}^{1} \cup S_{D_{1}, D_{0}}^{\prime}$ are linearly independent. Select any $u \notin S_{D_{0}, D_{1}}^{\prime}$ and apply Lemma 3.8 to $\zeta_{0}=u, \eta=t^{\prime} \oplus e_{j}, T=T_{D-1}^{0} \cup S_{D_{0}, D_{1}}^{\prime}$ to see that $\Gamma_{u} \notin \operatorname{span}\left(T_{D-1}^{0} \cup S_{D_{0}, D_{1}}^{\prime}\right)$. Note that when $\operatorname{wt}\left(\zeta_{0} \oplus \eta\right) \geq 4$, we do not yet know that $\Gamma_{\zeta_{0} \oplus e_{i *}}=\Gamma_{u \oplus e_{i}}$ lies outside $\operatorname{span}\left(T_{D-1}^{1} \cup S_{D_{1}, D_{0}}^{\prime}\right)$, that is, we do not know whether all the columns indexed by the set $S$ defined in Lemma 3.8 are linearly independent, but the second part of Lemma 3.8 says that we may still conclude that $\Gamma_{u} \notin \operatorname{span}\left(T_{D-1}^{0} \cup S_{D_{0}, D_{1}}^{\prime}\right)$ because the columns indexed by $S \backslash\left\{u \oplus e_{i^{*}}\right\}$ are linearly independent.

Lastly, apply Lemma 3.8 to $\zeta_{0}=u \oplus e_{i^{*}}, \eta=t \oplus e_{j}, T=$ $T_{D-1}^{1} \cup S_{D_{1}, D_{0}}^{\prime}$ to see that $\Gamma_{u \oplus e_{i^{*}}} \notin \operatorname{span}\left(T_{D-1}^{1} \cup S_{D_{1}, D_{0}}^{\prime}\right)$. Note that here we only need to invoke the first part of Lemma 3.8 we already know that $\Gamma_{\zeta_{0} \oplus e^{*}}=\Gamma_{u}$ lies outside $\operatorname{span}\left(T_{D-1}^{0} \cup S_{D_{0}, D_{1}}^{\prime}\right)$.

We are now ready to complete Inductive Step 1.

Proof of Inductive Step 1. As we remarked earlier, implication (15) for a fixed value of $\kappa$ implies implication (14) for that value of $\kappa$, so we just need to show that implication (14) also holds for $\kappa=K$. 
Suppose to the contrary that there exists pseudo-signature $\Gamma$ of rank $k$ such that $2^{K}+1 \leq k<2^{K+1}$. Without loss of generality, we may assume that for all clusters $Z \subsetneq$ $\{0,1\}^{m}, \operatorname{rank}\left(\Gamma_{Z}\right) \leq 2^{K}$; otherwise, replace $\Gamma$ by $\Gamma_{Z}$ for some small enough cluster $Z$ such that $\operatorname{rank}\left(\Gamma_{Z}\right) \geq 2^{K}+1$ and $\operatorname{rank}\left(\Gamma_{Z^{\prime}}\right) \leq 2^{K}$ for all subclusters $Z^{\prime} \subsetneq Z$. Furthermore, by Theorem 4.5 , we may assume $m>K+1$.

Lemma 4.6. If $Z=s+\left\{e_{p_{1}}, \ldots, e_{p_{K}}\right\}$ is a $(K, m)$-cluster of linearly independent columns in $\Gamma$, then any column $\Gamma_{t}$ for which $t_{i}=s_{i}$ for some $i \neq p_{1}, \ldots, p_{K}$ lies in the span of the columns indexed by $Z$.

Proof. If to the contrary there existed such a $\Gamma_{t}$ not lying in the span of $Z$ so that $t_{i}=s_{i}$ for some $i \neq p_{1}, \ldots, p_{K}$, then if $Z^{\prime}$ is the $(m-1, m)$-cluster of column indices $\zeta$ for which $\zeta_{i}=s_{i}, \Gamma_{Z^{\prime}}$ contains $t$ and all of $Z$ and thus has rank at least $2^{K}+1$, contradicting our assumption on the ranks of the proper subclusters of $\Gamma$.

By the inductive hypothesis, $\Gamma$ contains a $(K, m)$-cluster $Z$ of linearly independent columns $s+\left\{e_{p_{1}}, \ldots, e_{p_{K}}\right\}$. As $s$ is only uniquely defined modulo the bits in positions $p_{1}, \ldots, p_{K}$, we will leave those bits of $s$ unspecified for now.

Because $k>2^{K}$, there exists $t \notin Z$ for which all columns indexed by $Z \cup\{t\}$ are linearly independent. Moreover, by Lemma $4.6, t_{j}=\overline{s_{j}}$ for all $j \notin\left\{p_{1}, \ldots, p_{K}\right\}$. Let $Z^{\prime}$ denote the cluster $t+\left\{e_{p_{1}}, \ldots, e_{p_{K}}\right\}$. Set $s_{i}=t_{i}$ for $i \in\left\{p_{2}, \ldots, p_{K}\right\}$; we will set $s_{p_{1}}$ to be 0 or 1 depending on the parity of the number $m-K$ of bits outside of positions $p_{1}, \ldots, p_{K}$.

CASE 1. $m-K$ is even.

Set $s_{p_{1}}=t_{p_{1}}$ so that $s$ and $t$ have the same parity.

Claim 4.7. If $u \notin Z^{\prime}$ and $u_{i}=s_{i}$ for each $i \in\left\{p_{1}, \ldots, p_{K}\right\}$, then $\Gamma_{u}$ and $\Gamma_{s}$ are linearly dependent.

Proof. For each $i \in\left\{p_{1}, \ldots, p_{K}\right\}$ and $j \notin\left\{p_{1}, \ldots, p_{K}\right\}$, let $T_{i}$ denote the cluster of all column indices $u$ for which $u_{i}=s_{i}$, and let $T_{i}^{j}$ denote the cluster of all column indices $u$ for which $u_{i}=s_{i}$ and $u_{j}=s_{j}$. Let $Z_{i}=Z \cap T_{i}$; obviously $Z_{i} \subset T_{i}^{j} \subset T_{i}$.

Because $T_{i}^{j}$ is a cluster properly contained in $\{0,1\}^{m}$, we inductively know that $\operatorname{rank}\left(\Gamma_{T_{i}^{j}}\right) \leq 2^{K}$. And because $Z_{i} \subset$ $T_{i}^{j}, \operatorname{rank}\left(\Gamma_{T_{i}^{j}}\right) \geq 2^{K-1}$. But if $\operatorname{rank}\left(\Gamma_{T_{i}^{j}}\right) \geq 2^{K-1}+1$, then by inductive hypothesis (15) applied to $\Gamma_{T_{i}^{j}}$ for $\kappa=K-1$, $\operatorname{rank}\left(\Gamma_{T_{i}^{j}}\right) \geq 2^{K}$. In other words, $\operatorname{rank}\left(\Gamma_{T_{i}^{j}}\right)$ is either $2^{K-1}$ or $2^{K}$.

We will show that the latter is impossible. Suppose to the contrary that $\operatorname{rank}\left(\Gamma_{T_{i}^{j}}\right)=2^{K}$.

Then because $T_{i}^{j} \subset T_{i}$ and $\operatorname{rank}\left(\Gamma_{T_{i}}\right)=2^{K}=\operatorname{rank}\left(\Gamma_{T_{i}^{j}}\right)$, it follows that $\operatorname{span}\left(T_{i}\right)=\operatorname{span}\left(T_{i}^{j}\right)$. For any $u \in T_{i}^{j}, \Gamma_{u} \in$ $\operatorname{span}(Z)$ by Lemma 4.6, so

$$
\operatorname{span}(Z) \supset \operatorname{span}\left(T_{i}^{j}\right)=\operatorname{span}\left(T_{i}\right) .
$$

But $T_{i}$ contains $t$, and by definition $\Gamma_{t} \notin \operatorname{span}(Z)$, a contradiction.

We conclude that $\operatorname{rank}\left(\Gamma_{T_{i}^{j}}\right)=2^{K-1}$. Then because $Z_{i} \subset$ $T_{i}^{j}$ and $\operatorname{rank}\left(\Gamma_{Z_{i}}\right)=2^{K-1}=\operatorname{rank}\left(\Gamma_{T_{i}^{j}}\right)$, it follows that $\operatorname{span}\left(Z_{i}\right)=\operatorname{span}\left(T_{i}^{j}\right)$.
In particular, all columns indexed by $\bigcap_{k=1}^{K} T_{p_{k}}^{j}$ lie in

$$
\bigcap_{k=1}^{K} \operatorname{span}\left(Z_{p_{k}}\right)=\operatorname{span}(\{s\}) .
$$

Our choice of $j$ was arbitrary, so we get the desired claim.

From the above claim and the fact that we're assuming $m>K+1$, we conclude that $\Gamma_{s \oplus e_{j}}$ for any $j \notin\left\{p_{1}, \ldots, p_{K}\right\}$ lies in the span of $\Gamma_{s}$. But $s$ and $s \oplus e_{j}$ are of opposite parity, so by the parity condition, $\Gamma_{s \oplus e_{j}}=0$ for all such $j$. Applying Corollary 3.5 to $s$ and $t$, it follows that $\Gamma_{s}$ and $\Gamma_{t}$ are linearly dependent, a contradiction.

CAse 2. $m-K$ is odd.

Set $s_{p_{1}}=\overline{t_{p_{1}}}$ so that $s$ and $t$ have the same parity.

Claim 4.8. For any $u \in\{0,1\}^{m}$, if $u \notin Z^{\prime}$ and $u_{i}=s_{i}$ for all $i \in\left\{p_{2}, \ldots, p_{K}\right\}$, then:

1. If $u$ and $s$ have the same parity, then $\Gamma_{u}$ and $\Gamma_{s}$ are linearly dependent.

2. If $u$ and $s$ have the opposite parity, then $\Gamma_{u}$ and $\Gamma_{s \oplus e_{p_{1}}}$ are linearly dependent.

Proof. The proof is the same as that of Claim 4.7, the only subtlety being that $s$ and $t$ now only necessarily agree on bits $p_{2}, \ldots, p_{K}$. By the argument there, all columns indexed by $T_{i}^{j}$ lie in $\operatorname{span}\left(Z_{i}\right)$ for $i=p_{2}, \ldots, p_{K}$. In particular, for all $j \notin\left\{p_{1}, \ldots, p_{K}\right\}$, all columns indexed by $\bigcap_{k=2}^{K} S_{p_{k}}^{j}$ lie in $\bigcap_{k=2}^{K} \operatorname{span}\left(Z_{p_{k}}\right)=\operatorname{span}\left(\left\{s, s \oplus e_{p_{1}}\right\}\right)$.

So given $u \notin Z^{\prime}$, write $\Gamma_{u}=\alpha \Gamma_{s}+\beta \Gamma_{s \oplus e_{p_{1}}}$. If $u$ and $s$ have the same parity, $\beta=0$ by the parity condition, so $\Gamma_{u} \in \operatorname{span}(\{s\})$. If $u$ and $s$ have the opposite parity, $\alpha=0$ by the parity condition, so $\Gamma_{u} \in \operatorname{span}\left(\left\{s \oplus e_{p_{1}}\right\}\right)$.

Pick any $j \notin\left\{p_{1}, \ldots, p_{K}\right\}$ and define $s^{*}=s \oplus e_{j}$ and $t^{*}=t \oplus e_{j} . s^{*}$ and $t^{*}$ both satisfy the hypotheses of Claim 4.8 and have parity opposite to that of $s$, so by the latter case of Claim 4.8, they are both linearly dependent with $\Gamma_{s \oplus e_{p_{1}}}$. But $\Gamma_{s \oplus e_{p_{1}}} \neq 0$ because $s \oplus e_{p_{1}} \in Z$ and the columns indexed by $Z$ are linearly independent, so $\Gamma_{s^{*}}$ and $\Gamma_{t^{*}}$ are linearly dependent with each other.

To show $\Gamma_{s}$ and $\Gamma_{t}$ are linearly dependent, we wish to apply Corollary 3.6 to $s^{*}, t^{*}$, noting that $s^{*} \oplus t^{*}=e_{j} \oplus$ $\sum_{j^{\prime} \notin\left\{j, p_{2}, \ldots, p_{k}\right\}} e_{j}$.

For any $j^{\prime} \notin\left\{j, p_{2}, \ldots, p_{K}\right\}$, note that $s^{*} \oplus e_{j^{\prime}}$ and $t^{*} \oplus e_{j^{\prime}}$ both satisfy the hypotheses of Claim 4.8 and have the same parity as $s$, so by the former case of Claim 4.8, they are both linearly dependent with $\Gamma_{s}$. But $\Gamma_{s} \neq 0$ because $s \in Z$ and the columns indexed by $Z$ are linearly independent, so $\Gamma_{s^{*} \oplus e_{j^{\prime}}}$ and $\Gamma_{t^{*} \oplus e_{j^{\prime}}}$ are linearly dependent with each other.

Applying Corollary 3.6 to $s^{*}$ and $t^{*}$, it follows that $\Gamma_{s}$ and $\Gamma_{t}$ are linearly dependent, a contradiction.

\subsection{Existence of Cluster Submatrix}

Proof of Inductive Step 2. As in inductive step 1, we may assume without loss of generality that for all clusters $Z \subsetneq\{0,1\}^{m}, \operatorname{rank}\left(\Gamma_{Z}\right) \leq 2^{K}$. If $m=K+1$, then by the inductive hypothesis that (14) holds for $\kappa=K$, we're done. So suppose $m>K+1$. 
By the second part of the inductive hypothesis, implication (15) holds for $1 \leq \kappa \leq K-1$, so $\Gamma$ contains a $(K, m)$ cluster $Z$ of linearly independent columns $s+\left\{e_{p_{1}}, \ldots, e_{p_{K}}\right\}$.

As in inductive step 1, we can apply Lemma 4.6 to $Z$ to see that all columns outside the span of the columns indexed by $Z$ must be indexed by $Z^{\prime}=t+\left\{e_{p_{1}}, \ldots, e_{p_{K}}\right\}$, where $t=$ $\left(s \oplus \bigoplus_{i \neq p_{1}, \ldots, p_{K}} e_{i}\right)$. But $\left|Z^{\prime}\right|=|Z|=2^{K}$, and $\operatorname{rank}(\Gamma) \geq$ $2^{K+1}$ by implication (14) for $\kappa=K$, so the columns indexed by $Z \cup Z^{\prime}$ are linearly independent. Because $m>K+1$, there exist columns not indexed by either $Z$ or $Z^{\prime}$, and by Lemma 4.6 applied once to $Z$ and once to $Z^{\prime}$, these columns are in both $\operatorname{span}(Z)$ and $\operatorname{span}\left(Z^{\prime}\right)$ and thus must be zero.

If $s$ and $t$ are of the same parity, apply Corollary 3.5 to $s$ and $t$ to find that $\Gamma_{s}$ and $\Gamma_{t}$ are linearly dependent, a contradiction.

If $s$ and $t$ are of opposite parity, apply Corollary 3.6 to $s \oplus e_{j}$ and $t \oplus e_{j} \oplus e_{p_{1}}$ for any $j \notin\left\{p_{1}, \ldots, p_{K}\right\}$ to find that $\Gamma_{s}$ and $\Gamma_{t \oplus e_{p_{1}}}$ are linearly dependent, a contradiction.

\section{GROUP PROPERTY OF STANDARD SIG- NATURES}

We will now prove the following generalization of the group property result over domain size 4 due to Cai and Fu (Theorem $5.5,[5])$ :

THEOREM 5.1. If $G$ is a generator matchgate of arity $K n$ with standard signature $\underline{G}$, and $\operatorname{rank}(\underline{G}(t))=2^{K}$ for some $t$, then there exists a recognizer matchgate of arity $K n$ such that $\underline{G}(t) \underline{R}(t)=I_{2}$.

Roughly, we invoke Theorem 4.3 to obtain a full-rank $K$ cluster submatrix $G^{\prime}$ of $\underline{G}(t)$ with column indices belonging to cluster $\zeta+\left\{e_{p_{1}}, \ldots, e_{p_{K}}\right\}$. Assume without loss of generality that $\zeta_{p_{i}}=0$ for all $i \in[K]$. We will show that the matrix obtained by replacing $G^{\prime}$ in $\underline{G}(t)$ with $\left(G^{\prime}\right)^{-1}$ and the remaining entries with zeroes is the standard signature of some arity- $K n$ recognizer.

We first fix some notation. Denote $\underline{G}(t)$ by $\Gamma$. Suppose that nodes $p_{1}<\cdots<p_{m} \in[K n]$ belong to blocks before the $t$-th, and nodes $p_{m+1}<\cdots<p_{K} \in[K n]$ belong to blocks after the $t$-th. For expository purposes, we wish to use a particular permutation $\left(q_{1}, \ldots, q_{K}\right)$ of $\left(p_{1}, \ldots, p_{K}\right)$, so for $i \leq m$, let $q_{i}=p_{m-i+1}$, and for $i>m$, let $q_{i}=p_{K+m-i+1}$ (see Figure 1). If the column indices of $\Gamma$ are of the form $i_{1} \cdots i_{K(n-1)}$, those of $G^{\prime}$ are of the form $i_{p_{1}} \cdots i_{p_{K}}$.

In [13], Li and Xia gave a constructive proof that in the character theory of matchgates, the $2^{K} \times 2^{K}$ character matrices of invertible $K$-input, $K$-output matchgates form a group under matrix multiplication. One can check that their construction carries over to show that the $2^{K} \times 2^{K}$ standard signatures of such matchgates likewise form a group, but unfortunately this is not enough to prove Theorem 5.1, as $G^{\prime}$ alone is merely a pseudo-signature and may not be realizable as the standard signature of a $K$-input, $K$-output matchgate. That said, Theorem 5.1 can still be proved with minor modifications to $\mathrm{Li}$ and Xia's approach.

We begin with a toy example motivating the notation in the previous paragraphs. Suppose that for each $i \in[K]$, there exists an edge of weight 1 such that the $i$-th external node in block $t$ and external node $q_{i}$ are both incident only to this edge. Note that in this case, $G^{\prime}$ is a symmetric permutation matrix and thus equal to its own inverse.
We can easily construct a recognizer $R$ out of $G$ for which $\underline{G}(t) \underline{R}(t)=I_{2} \mathrm{~K}$ as follows. Remove all non-external nodes of $G$, as well as all edges incident to nodes outside of block $t$ and nodes $q_{1}, \ldots, q_{K}$. For external node $i$ outside of block $t$ such that $i \notin\left\{q_{1}, \ldots, q_{K}\right\}$, if $\zeta_{i}=0$, attach a distinct edge of weight 1 to node $i$ and designate the other endpoint of the edge as the $i$ th external node of $R$; if $\zeta_{i}=1$, attach a distinct path graph of length 2 consisting of two edges of weight 1 , and denote the other endpoint of the path as the $i$ th external node of $R$. By construction, in the $2^{K(n-1)} \times 2^{K}$ matrix $\underline{R}(t)$, the submatrix indexed by rows $q_{1}, \ldots, q_{K}$ is equal to $G^{\prime}$, and all other entries are zero. Because $G^{\prime}=\left(G^{\prime}\right)^{-1}$, $\underline{G}(t) \underline{R}(t)=I_{2 K}$ as desired.

See Figure 1 for an example of this construction.

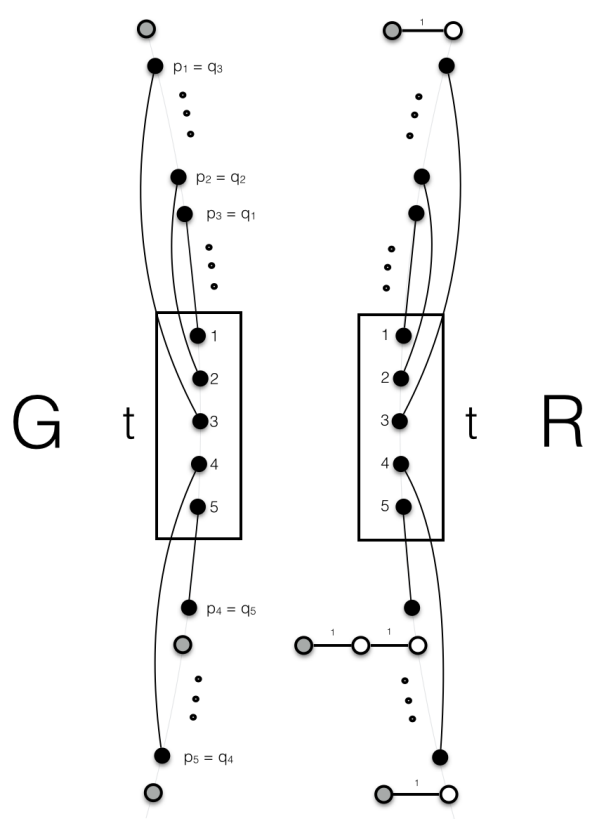

Figure 1: Toy example of $G$ reduced at $i=1, \ldots, K$. Here, $\ell=5, K=5, m=3$. Black nodes in $G$ denote nodes $1, \ldots, K$ in block $t$ and nodes $q_{1}, \ldots, q_{K}$. External nodes of $G$ and $R$ are shown in black/gray.

Definition 5.2. For $i \in[K]$, if $i \leq m$ (resp. $i>m$ ), $G$ is reduced at $i$ if there exists an edge of weight 1 in $G$ connecting the $i$-th external node in block $t$ and external node $q_{i}$ such that these two nodes are both incident only to that edge.

To prove Theorem 5.1, it is enough to reduce to the special case of the toy example above where $\Gamma$ is realizable by a generator $G$ reduced at all $i \in[K]$. The rest of this section will be dedicated to proving the following:

LEMMA 5.3. If $\Gamma$ is the standard signature of a generator of arity $K n$ reduced at $1, \ldots, i$, there exist nonsingular $K$ input, $K$-output transducers $L_{1}, \ldots, L_{r}$ and $K(n-1)$-input, $K(n-1)$-output transducers $R_{1}, \ldots, R_{s}$ such that $\underline{L_{r}} \cdots \underline{L_{1}}$. $\Gamma \cdot \underline{R_{1}} \cdots \underline{R_{s}}$ is the standard signature of a generator reduced at $\overline{1, \ldots, i+1}$. 
We first give a sufficient characterization of standard signatures of matchgates reduced at $1, \ldots, i$ in terms of the entries of their standard signatures.

The following terminology is borrowed from [13].

Definition 5.4. Let $M$ be any $2^{r} \times 2^{c}$ matrix whose rows and columns are indexed by $\sigma \in\{0,1\}^{r}$ and $\tau \in\{0,1\}^{c}$ respectively. $\Gamma_{\tau}^{\sigma}$ is an edge entry of $M$ iff $r+c-2 \leq \operatorname{wt}(\sigma)+$ $\operatorname{wt}(\tau)<r+c$.

LEMMA 5.5. $\Gamma$ is the standard signature of a generator $G$ that is reduced at $i$ if $\Gamma$ satisfies the following:

$$
\begin{aligned}
& \text { 1. }\left(G^{\prime}\right)_{1^{K}}^{1^{K}}=\left(G^{\prime}\right)_{1^{K} \oplus 1_{q_{i}}^{K} \oplus e_{i}}=1 \text {. } \\
& \text { 2. }\left(G^{\prime}\right)_{\tau}^{\sigma}=0 \text { for all other edge entries of } G^{\prime} \text { for which } \\
& \sigma \in\left\{1^{K}, 1^{K} \oplus e_{i}\right\} \text { or } \tau \in\left\{1^{K}, 1^{K} \oplus e_{q_{i}}\right\} \text {. }
\end{aligned}
$$

To show this, it suffices to prove the following useful consequence of the matchgate identities, first observed in [4] and translated below to our setting of standard signatures in matrix form.

Lemma 5.6 (Theorem 4.2, [4]). If $\left(G^{\prime}\right)_{1 K}^{1^{K}} \neq 0$, the entries of $G^{\prime}$ are uniquely determined by $\left(G^{\prime}\right)_{1 K}^{1^{K}}$ and the edge entries of $G^{\prime}$.

Proof. Assume that these entries uniquely determine all entries $\Gamma_{\tau}^{\sigma}$ for which $\operatorname{wt}(\sigma)+\operatorname{wt}(\tau) \geq m$ for some $m \leq$ $n-2$. We proceed by downward induction on $m$ (by the parity condition, if $m$ is even, the case of $m+1$ follows immediately from that of $m$ ). Then for $\sigma, \tau \in\{0,1\}^{K}$ such that $\operatorname{wt}(\sigma)+\operatorname{wt}(\tau)=m-2$, apply (2) from Theorem 3.1 to $\sigma:=\sigma, \zeta:=\tau, \tau:=1^{K}$, and $\eta:=1^{K}$. One can check that the resulting identity consists of $\left(G^{\prime}\right)_{\tau}^{\sigma} \cdot\left(G^{\prime}\right)_{1}^{1^{K}}$ and terms which have already been uniquely determined by the inductive hypothesis, so because $\left(G^{\prime}\right)_{1^{K}}^{1^{K}} \neq 0$, we conclude that $\left(G^{\prime}\right)_{\tau}^{\sigma}$ is also uniquely determined.

OBSERVATION 2. If $\Gamma$ is the standard signature of a generator reduced at $i,\left(G^{\prime}\right)_{\tau \oplus e_{q_{i}}}^{\sigma \oplus e_{i}}=\left(G^{\prime}\right)_{\tau}^{\sigma}$, and if $\sigma_{i} \neq \tau_{q_{i}}$, $\left(G^{\prime}\right)_{\tau}^{\sigma}=0$.

Proof. By hypothesis, external node $i$ of block $t$ and external node $q_{i}$ are only connected to each other. If $\sigma_{i} \neq \tau_{q_{i}}$, $\left(G^{\prime}\right)_{\tau}^{\sigma}$ counts the number of perfect matchings of $\Gamma$ where, among other conditions, exactly one of these two nodes is removed, and no such matching exists. On the other hand, if $\sigma_{i}=\tau_{q_{i}}$, then $\left(G^{\prime}\right)_{\tau}^{\sigma}$ and $\left(G^{\prime}\right)_{\tau \oplus e_{q_{i}}}^{\sigma \oplus e_{i}}$ count the number of perfect matchings in which, among other conditions, both of these two nodes are removed, or neither is. The number of perfect matchings in either scenario is the same.

Let $G_{i}^{\prime}$ be the $2^{K-i} \times 2^{K-i}$ submatrix of $G^{\prime}$ consisting of entries $\left(G^{\prime}\right)_{\tau}^{\sigma}$ for which $\sigma_{j}=0$ for $j=1, \ldots, i$ and $\tau_{j}=$ 0 for $j=q_{1}, \ldots, q_{i}$. If the row and column indices of $G^{\prime}$ are of the form $i_{1} \cdots i_{K}$ and $i_{p_{1}} \cdots i_{p_{K}}$ respectively, the row and column indices of $G_{i}^{\prime}$ are of the form $i_{i+1} \cdots i_{K}$ and $i_{p_{i+1}} \cdots i_{p_{K}}$ respectively. When referring to the row (resp. column) of $G^{\prime}$ containing a row $i_{i+1} \cdots i_{K}$ (resp. column $i_{p_{i+1}} \cdots i_{p_{K}}$ ) of $G_{i}^{\prime}$, we use the notation $0^{i} \circ i_{i+1} \cdots i_{K}$ (resp. $\left.0^{i} \circ i_{p_{i+1}} \cdots i_{p_{K}}\right)$ to denote its index in $G^{\prime}$. For example, column $0^{i} \circ 1^{K-i}$ of $G^{\prime}$ is the column of $G^{\prime}$ indexed by $\sigma \in$ $\{0,1\}^{K}$ for which $\sigma_{q_{1}}=\cdots=\sigma_{q_{i}}=0$ and $\sigma_{q_{i+1}}=\cdots=$ $\sigma_{q_{K}}=1$, and this contains column $1^{K-i}$ of $G_{i}^{\prime}$.
COROLlary 5.7. If $\Gamma$ is the standard signature of a generator that is reduced at $1, \ldots, i$, then $\Gamma$ is the standard signature of a generator reduced at $1, \ldots, i+1$ if $\Gamma$ satisfies the following:

$$
\text { 1. }\left(G_{i}^{\prime}\right)_{1^{K-i}}^{1^{K-i}}=\left(G_{i}^{\prime}\right)_{1^{K-i} \oplus e_{q_{i+1}}}^{1^{K-i} \oplus e_{i+1}}=1
$$

2. $\left(G_{i}^{\prime}\right)_{\tau}^{\sigma}=0$ for all other edge entries of $G_{i}^{\prime}$ such that $\sigma \in\left\{1^{K-i}, 1^{K-i} \oplus e_{i+1}\right\}$ or $\tau \in\left\{1^{K-i}, 1^{K-i} \oplus e_{q_{i+1}}\right\}$.

Proof. Apply Observation 2 to each of $1, \ldots, i$, and invoke Lemma 5.5.

Proof of Lemma 5.3 (Sketch). Our strategy and constructions are very similar to those in [13]. We want to execute the transformation $\Gamma=\Gamma^{(0)} \Rightarrow \Gamma^{(1)} \Rightarrow \Gamma^{(2)} \Rightarrow \Gamma^{(3)} \Rightarrow$ $\Gamma^{(4)}$ outlined below.

1. $\left(\Gamma^{(0)} \Rightarrow \Gamma^{(1)}\right)$ : Turn the entry indexed by $\left(1^{K-i}, 1^{K-i}\right)$ in $G_{i}^{\prime}$ to 1 .

2. $\left(\Gamma^{(1)} \Rightarrow \Gamma^{(2)}\right)$ : Turn edge entries of $G_{i}^{\prime}$ in row or column $1^{K-i}$ to 0 .

3. $\left(\Gamma^{(2)} \Rightarrow \Gamma^{(3)}\right)$ : Turn entry $\left(1^{K-i} \oplus e_{i+1}, 1^{K-i} \oplus e_{q_{i+1}}\right)$ in $G_{i}^{\prime}$ to 1 .

4. $\left(\Gamma^{(3)} \Rightarrow \Gamma^{(4)}\right)$ : Turn all other edge entries in $G_{i}^{\prime}$ in row $1^{K-i} \oplus e_{i+1}$ or column $1^{K-i} \oplus e_{q_{i+1}}$ to zero.

We need not care what these transformations do to entries outside of $G^{\prime}$, but we must ensure they preserve the fact that $\Gamma$ is the standard signature of a generator reduced at $1, \ldots, i$. To do this, for each matrix $M$ by which we left- or rightmultiply $\Gamma$, if $\sigma$ does not index a row (resp. column) of $G_{i}^{\prime}$, the only nonzero entry of $M$ in row (resp. column) $\sigma$ will be 1 in column (resp. row) $\sigma$.

In each step $j$, we will for convenience refer to $\Gamma^{(j-1)}$ as $\Gamma$.

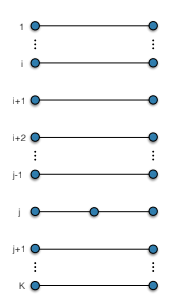

(a) $C_{j}$

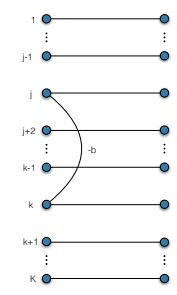

(b) $L_{3}^{j, k}$

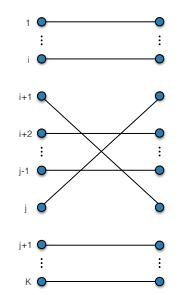

(c) $L_{4}$

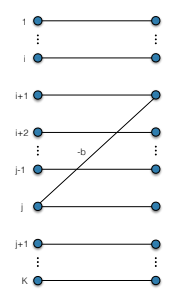

(d) $L_{5}^{j}$
Figure 2: Transducers realizing row/column operations in steps 1-4

SteP 1. $\left(\Gamma^{(0)} \Rightarrow \Gamma^{(1)}\right)$ : Turn the entry in $G_{i}^{\prime}$ with index $\left(1^{K-i}, 1^{K-i}\right)$ to 1 .

We first show how to move a nonzero entry $c:=\left(G_{i}^{\prime}\right)_{\tau^{*}}^{\sigma^{*}}$ of $G_{i}^{\prime}$ into entry $\left(1^{K-i}, 1^{K-i}\right)$ of $G_{i}^{\prime}$.

For each $j$ for which $i<j \leq K$, we would like a $2^{K} \times 2^{K}$ standard signature $C_{j}$ such that left-multiplication of $\Gamma$ by $C_{j}$ interchanges row $\sigma$ in $G^{\prime}$ with $\sigma \oplus e_{j}$ for all $\sigma \in\{0,1\}^{K}$, and a $2^{K(n-1)} \times 2^{K(n-1)}$ standard signature $D_{j}$ such that right-multiplication of $\Gamma$ by $D_{j}$ interchanges column $\tau$ in $G^{\prime}$ 
with $\tau \oplus e_{q_{j}}$. We could then define $L_{1}=\prod_{j: \sigma_{j}^{*}=0} C_{j}$ and $R_{1}=\prod_{j: \tau_{j}^{*}=0} D_{j}$, and $L_{1} \cdot \Gamma R_{1}$ would have nonzero entry $c$ at index $\left(1^{K-i}, 1^{K-i}\right)$ of $G_{i}^{\prime}$ and still be the standard signature of a matchgate reduced at $1, \ldots, i$.

$C_{j}$ (resp. $D_{j}$ ) is the permutation matrix whose only nonzero entry in row $\sigma \in\{0,1\}^{K}$ (resp. $\sigma \in\{0,1\}^{K(n-1)}$ ) is 1 in column $\sigma \oplus e_{j}$ (resp. column $\sigma \oplus e_{q_{j}}$ ) if $G_{i}^{\prime}$ contains entries from $\Gamma^{\sigma}$ (resp. $\Gamma_{\sigma}$ ), and 1 in column $\sigma$ otherwise. $C_{j}$ and $D_{j}$ are certainly nonsingular.

To construct the $K$-input, $K$-output transducer realizing $C_{j}$ as a standard signature, begin with a $(K, K)$-bipartite graph where for every $\nu \neq j$, left node $\nu$ and right node $\nu$ are connected by an edge of weight 1 . Add an extra vertex between left node $j$ and right node $j$, and draw a path of length two connecting these three vertices, where both edges of the path have weight 1 . This construction is shown in Figure 2a.

The $K(n-1)$-input, $K(n-1)$-output transducer realizing $D_{j}$ as a standard signature is similarly constructed, the only difference being that the bipartite graph has left and right vertex sets of size $K(n-1)$, and the path of length two is drawn between left node $q_{j}$ and right node $q_{j}$.

Next, we want to scale all of the entries of $L_{1} \Gamma R_{1}$ by a factor of $1 / c$, so define $L_{2}(c)$ to be the diagonal matrix whose entry at index $1^{K-i}$ is $1 / c$ and whose entries at all other indices are 1 . Obviously $L_{2}(c)$ is nonsingular and satisfies the matchgate identities (2). We take $\Gamma^{(1)}=L_{2}(c) L_{1} \Gamma^{(0)} R_{1}$.

For brevity, we omit the remaining steps (see the full version of this paper for the remaining steps); the matchgates used in all four steps are shown in Figure 5. The only subtlety in the remaining steps is that the corresponding matchgates are not planar. But by Lemmas A.1, A.2, and A.3 in Appendix A, there exist planar matchgates whose standard signatures agree with those of the matchgates in Figure 5 up to a factor of -1 in certain inconsequential entries.

\section{REDUCING TO DOMAIN SIZE $2^{K}$}

In this section we use Theorem 4.1 to reduce proving a basis collapse theorem over all domain sizes to proving one over domain sizes $2^{K}$. The result we will prove is the following generalization of the main result in [11] whose strategy we follow.

Theorem 6.1. Suppose Theorem 1.1 has been proven for domain size $r$. If recognizer signatures $R_{1}, \ldots, R_{a}$ and generator signatures $G_{1}, \ldots, G_{b}$ on domain size $k>r$ belonging to matchgrid $\Omega$ are simultaneously realizable on a $2^{\ell} \times k$ basis $M$ of rank $r$ and $R_{1}$ is of full rank, then there exists a basis $M^{\prime}$ of size at most $\left\lfloor\log _{2} r\right\rfloor$ on which they are simultaneously realizable.

We'll need some preliminaries before we can prove this. Express $M$ as $\left(\begin{array}{llll}\alpha_{1} & \alpha_{2} & \cdots & \alpha_{k}\end{array}\right)$ where each $\alpha_{i}$ is a $2^{\ell}$ dimensional column vector. Let $i_{1}, \ldots, i_{r} \in[k]$ be column indices of $M$ for which $M^{i_{1} \cdots i_{r}}:=\left(\begin{array}{llll}\alpha_{i_{1}} & \alpha_{i_{2}} & \cdots & \alpha_{i_{r}}\end{array}\right)$ is of full rank. Define sub-signature $R^{i_{1} \cdots i_{r}}$ to consist of entries $\left(R_{j_{1} \cdots j_{n}}\right)$ of $R$ ranging over all $j_{1}, \ldots, j_{n} \in\left\{i_{1}, \ldots, i_{r}\right\} \subset[k]$. We can define the sub-signature $G^{i_{1} \cdots i_{r}}$ of a generator analogously. Equivalently,

$$
R^{i_{1} \cdots i_{r}}=\underline{R}\left(M^{i_{1} \cdots i_{r}}\right)^{\otimes n}
$$

LEMMA 6.2. For a recognizer $R$ realizable on the basis $M$, if there exists $t$ for which $\operatorname{rank}(R(t)) \geq r$, then

$$
\operatorname{rank}\left(R^{i_{1} \cdots i_{r}}(t)\right)=r
$$

Proof. By Lemma 2.14, $R(t)=\left(M^{T}\right)^{\otimes(n-1)} \underline{R}(t) M$, so $\operatorname{rank}(\underline{R}(t)) \geq r$. By (16) and Lemma 2.13, $R^{i_{1} \cdots i_{r}}(t)=$ $\left(\left(M^{i_{1} \cdots i_{r}}\right)^{T}\right)^{\otimes(n-1)} \underline{R}(t) M^{i_{1} \cdots i_{r}}$, so $\operatorname{rank}\left(R^{i_{1} \cdots i_{r}}(t)\right)=r$.

For such a recognizer $R$, define for each $w \in[k]$ a $n k^{n-1}$ dimensional column vector $b_{w}$ by

$$
b_{w}=\left(R_{w 1 \cdots 11} \cdots R_{w k \cdots k k} \cdots \cdots R_{11 \cdots 1 w} \cdots R_{k k \cdots k w}\right)^{T}
$$

and define $A_{i_{1} \cdots i_{r}}$ to be the $n k^{(n-1)} \times r$ matrix whose $j$ th column is $b_{i_{j}}$.

OBSERVATion 3. $\operatorname{rank}\left(A_{i_{1} \cdots i_{r}}\right)=r$.

PROOF. $R^{i_{1} \cdots i_{r}}(t)$ is a submatrix of $A_{i_{1} \cdots i_{r}}$ and already has rank $r$ by Lemma 6.2.

OBSERVATION 4. As $M$ has rank $r$, every column $\alpha_{w}$ can be expressed as a linear combination $\sum_{j=1}^{r} x_{w}^{i_{j}} \alpha_{i_{j}}$.

Denote the $r \times k$ matrix $\left(x_{w}^{i_{j}}\right)$ of these coefficients by $X_{i_{1} \cdots i_{r}}$.

Lemma 6.3. For each $w \in[k], A_{i_{1} \cdots i_{r}} X=b_{w}$ has the unique solution $X=\left(\begin{array}{lll}x_{w}^{i_{1}} & \cdots & x_{w}^{i_{r}}\end{array}\right)^{T}$.

Proof. A solution for $X$ exists and is unique because $\operatorname{rank}\left(A_{i_{1} \cdots i_{r}}\right)=r$ by Observation 3 . To check that the purported solution for $X$ is correct, pick any entry of $b_{w}$, call it $R_{j_{1} \cdots j_{t-1} w j_{t} \cdots j_{n}}$ of $b_{w}$. By definition of recognizer signatures,

$$
\begin{aligned}
& R_{j_{1} \cdots j_{t-1} w j_{t} \cdots j_{n}} \\
= & \left\langle\underline{R}, \alpha_{j_{1}} \otimes \cdots \otimes \alpha_{j_{t-1}} \otimes \alpha_{w} \otimes \alpha_{j_{t+1}} \otimes \cdots \otimes \alpha_{j_{n}}\right\rangle \\
= & \left\langle\underline{R}, \alpha_{j_{1}} \otimes \cdots \otimes \alpha_{j_{t-1}} \otimes\left(\sum_{j=1}^{r} x_{w}^{i_{j}} \alpha_{i_{j}}\right) \otimes \alpha_{j_{t+1}} \otimes \cdots \otimes \alpha_{j_{n}}\right\rangle \\
= & \sum_{j=1}^{r} x_{w}^{i_{j}} R_{j_{1} \cdots j_{t-1} i_{j} j_{t+1} \cdots j_{n}} .
\end{aligned}
$$

Here $\langle\cdot, \cdot\rangle$ denotes the inner product.

The content of Lemma 6.3 is that to any such $R$ we can get a matrix $X_{i_{1} \cdots i_{r}}$ without needing to know the actual basis $M$ realizing $R$.

LemmA 6.4. If $\operatorname{rank}\left(R_{1}(t)\right) \geq r$ for some $t$, then recognizers $R_{1}, \ldots, R_{a}$ are simultaneously realizable on some basis of rank $r$ iff the following conditions hold:

1. $\operatorname{rank}\left(R_{1}^{i_{1} \cdots i_{r}}(t)\right)=r$ for some $i_{1}, \ldots, i_{r} \in[k]$.

2. There exists a unique $r \times k$ matrix $X_{i_{1} \cdots i_{r}}=\left(x_{w}^{i_{j}}\right)$ such that $A_{i_{1} \cdots i_{r}} X=b_{w}$ possesses the solution $X=$ $\left(\begin{array}{lll}x_{w}^{i_{1}} & \cdots & x_{w}^{i_{r}}\end{array}\right)^{T}$ for each $w \in[k]$.

3. There exists a $2^{\ell} \times r$ basis $M_{(r)}$ such that the $R_{j}^{i_{1} \cdots i_{r}}$ are simultaneously realizable on $M_{(r)}$ for all $j \in[a]$.

4. $R_{j}=R_{j}^{i_{1} \cdots i_{r}} X_{i_{1} \cdots i_{r}}^{\otimes n}$ for all $j \in[a]$. 
Proof. Suppose $R_{1}, \ldots, R_{a}$ are simultaneously realizable are some basis $M$. Conditions 1 and 2 follow from Lemma 6.2 and Lemma 6.3 respectively. Take $M_{(r)}$ to be $M^{i_{1} \cdots i_{r}}$, and condition 3 follows from the definition of sub-signature. By Observation $4, X_{i_{1} \cdots i_{r}}$ satisfies $M_{(r)} X_{i_{1} \cdots i_{r}}=M$, so $R_{j}=$ $\underline{R}_{j} M^{\otimes n}=\underline{R}_{j} M_{(r)}^{\otimes n} X_{i_{1} \cdots i_{r}}^{\otimes n}=R_{j}^{i_{1} \cdots i_{r}} X_{i_{1} \cdots i_{r}}^{\otimes n}$, and condition 4 follows.

Conversely, suppose conditions 1-4 hold. Condition 3 tells us that there is some $M_{(r)}$ for which $R_{j}^{i_{1} \cdots i_{r}}=\underline{R}_{j}\left(M_{(r)}\right)^{\otimes n}$ for all $j \in[a]$. Then

$$
R_{j}=\underline{R}_{j}\left(M_{(r)}\right)^{\otimes n} X_{i_{1} \cdots i_{r}}^{\otimes n}=\underline{R}_{j}\left(M_{(r)} X_{i_{1} \cdots i_{r}}\right)^{\otimes n},
$$

so the signatures $R_{1}, \ldots, R_{a}$ are simultaneously realizable on $M:=M_{(r)} X_{i_{1}, \ldots, i_{r}}$.

THEOREM 6.5. If recognizer signatures $R_{1}, \ldots, R_{a}$ and generator signatures $G_{1}, \ldots, G_{b}$ in matchgrid $\Omega$ are simultaneously realizable on a basis of rank $r$ and there exists $t$ for which $\operatorname{rank}\left(R_{1}(t)\right) \geq r$, then there exist recognizer signatures $\check{R}_{1}, \ldots, \check{R}_{a}$ and generator signatures $\check{G}_{1}, \ldots, \check{G}_{b}$ in matchgrid $\Omega^{\prime}$ over domain size $r$ that are simultaneously realizable on a $2^{\ell} \times r$ basis $M_{(r)}$. Furthermore,

$$
\operatorname{Holant}(\Omega)=\operatorname{Holant}\left(\Omega^{\prime}\right) \text {. }
$$

Proof. We first construct $\check{R}_{1}, \ldots, \check{R}_{a}, \check{G}_{1}, \ldots, \breve{G}_{b} . X_{i_{1}, \ldots, i_{r}}$ obtained from $R_{1}$ via Lemma 6.3 has rank $r$, so let $X_{i_{1} \cdots i_{r}}^{\prime}$ be the $k \times k$ invertible matrix for which $X_{i_{1} \cdots i_{r}} X_{i_{1} \cdots i_{r}}^{\prime}=$ $\left(I_{r} \mid \mathbf{0}_{r \times(k-r)}\right)$, where $I_{r}$ is the $r \times r$ identity matrix and $\mathbf{0}_{r \times(k-r)}$ denotes the $r \times(k-r)$ matrix consisting solely of zeroes. For each $j \in[a]$, let $R_{j}^{\prime}=R_{j}\left(X_{i_{1}, \ldots, i_{r}}^{\prime}\right)$, and let $\check{R}_{j}$ be the sub-signature $\left(R_{j}^{\prime}\right)^{1 \cdots r}$. Likewise, for each $j \in[b]$, let $G_{j}^{\prime}$ be defined by $G_{j}=\left(X_{i_{1} \cdots i_{r}}^{\prime}\right)^{\otimes n} G_{j}^{\prime}$, and let $\check{G}_{j}$ be the sub-signature $\left(G_{j}^{\prime}\right)^{1 \cdots r}$.

Claim 6.6. For all $j, \check{R}_{j}=R_{j}^{i_{1} \cdots i_{r}}$ and $\check{G}_{j}=G_{j}^{i_{1} \cdots i_{r}}$.

Proof. We need to check that

$$
\begin{gathered}
\check{R}_{j}=\underline{R}_{j}\left(M^{i_{1} \cdots i_{r}}\right)^{\otimes n} \\
\left(M^{i_{1} \cdots i_{r}}\right)^{\otimes n} \check{G}_{j}=\underline{G}_{j} .
\end{gathered}
$$

Indeed,

$$
\begin{aligned}
R_{j}^{\prime} & =\underline{R}_{j} M^{\otimes n}\left(X_{i_{1} \cdots i_{r}}^{\prime}\right)^{\otimes n} \\
& =\underline{R}_{j}\left(M^{i_{1} \cdots i_{r}}\right)^{\otimes n} X_{i_{1} \cdots i_{r}}^{\otimes n}\left(X_{i_{1} \cdots i_{r}}^{\prime}\right)^{\otimes n} \\
& =\underline{R}_{j}\left(M^{i_{1} \cdots i_{r}} \mid \mathbf{0}_{r \times(k-r)}\right)^{\otimes n},
\end{aligned}
$$

proving (19). Similarly,

$$
\begin{aligned}
\underline{G}_{j} & =M^{\otimes n} G_{j} \\
& =M^{\otimes n}\left(X_{i_{1} \cdots i_{r}}^{\prime}\right)^{\otimes n} G_{j}^{\prime} \\
& =\left(M^{i_{1} \cdots i_{r}} \mid \mathbf{0}_{r \times(k-r)}\right)^{\otimes n} G_{j}^{\prime},
\end{aligned}
$$

proving (20).

We conclude that $\check{R}_{1}, \ldots, \check{R}_{a}, \check{G}_{1}, \ldots, \check{G}_{b}$ are simultaneously realizable on the basis $M_{(r)}:=M^{i_{1} \cdots i_{r}}$.

To check that the Holants agree, note that if $R_{1}^{\prime}, \ldots, R_{a}^{\prime}$, $G_{1}^{\prime}, \ldots, G_{b}^{\prime}$ lie in a corresponding matchgrid $\Omega^{\prime \prime}$, Holant $(\Omega)=$ Holant $\left(\Omega^{\prime \prime}\right)$ because we're just applying a basis change from
$M$ to $M X_{i_{1} \cdots i_{r}}^{\prime}$. And Holant $\left(\Omega^{\prime}\right)=\operatorname{Holant}\left(\Omega^{\prime \prime}\right)$ because the operation of taking sub-signatures does not lose any information in this case, i.e. $\left(R_{j}^{\prime}\right)_{\sigma}=0$ for all $\sigma \in[k]^{n} \backslash[r]^{n}$.

For the next two results, suppose recognizer signatures $R_{1}, \ldots, R_{a}$ and generator signatures $G_{1}, \ldots, G_{b}$ in matchgrid $\Omega$ are simultaneously realizable on a basis of rank $r$ and there exists $t$ for which $\operatorname{rank}\left(R_{1}(t)\right) \geq r$.

THEOREM 6.7. If the recognizer signatures $\check{R}_{1}, \ldots, \check{R}_{a}$ and generator signatures $\check{G}_{1}, \ldots, \check{G}_{b}$ constructed in Theorem 6.5 are also simultaneously realizable on a $2^{\ell^{\prime}} \times r$ basis $M_{(r)}^{\prime}$ of rank $r$, then recognizer signatures $R_{1}, \ldots, R_{a}$ and generator signatures $G_{1}, \ldots, G_{r}$ are simultaneously realizable on the $2^{\ell^{\prime}} \times k$ basis $M_{(r)}^{\prime} X_{i_{1} \cdots i_{r}}$, where $X_{i_{1} \cdots i_{r}}$ is obtained from $R_{1}$ by Lemma 6.3.

Proof.

$$
R_{j}=R_{j}^{i_{1} \cdots i_{r}} X_{i_{1} \cdots i_{r}}^{\otimes n}=\check{R}_{j} X_{i_{1} \cdots i_{r}}^{\otimes n}=\underline{R}\left(M_{(r)}^{\prime} X_{i_{1} \cdots i_{r}}\right)^{\otimes n},
$$

where the first equality holds by condition 4 of Lemma 6.4, the second by Claim 6.6, the third by definition of $M_{(r)}^{\prime}$. Likewise, because

$$
\check{G}_{j}=\left(I_{r} \mid \mathbf{0}_{r \times(k-r)}\right) G_{j}^{\prime}=X_{i_{1} \cdots i_{r}} X_{i_{1} \cdots i_{r}}^{\prime} G_{j}^{\prime},
$$

we have that

$$
\underline{G}_{j}=\left(M_{(r)} X_{i_{1} \cdots i_{r}}\right)^{\otimes n} X_{i_{1} \cdots i_{r}}^{\prime \otimes n} G_{j}^{\prime}=\left(M_{(r)} X_{i_{1} \cdots i_{r}}\right)^{\otimes n} G_{j},
$$

so we conclude that $R_{1}, \ldots, R_{a}, G_{1}, \ldots, G_{b}$ are indeed simultaneously realizable on $M_{(r)} X_{i_{1} \cdots i_{r}}$.

We are now ready to prove Theorem 6.1.

Proof of Theorem 6.1. By Theorem 6.5, we know that $\check{R}_{1}, \ldots, \check{R}_{a}$ and $\breve{G}_{1}, \ldots, \breve{G}_{b}$ on domain size $r$ are simultaneously realizable on a $2^{\ell} \times r$ basis.

By definition, $\check{R}_{1}=R_{1}^{i_{1} \cdots i_{r}}$, and because $R_{1}$ was assumed to be full-rank, Lemma 6.2 tells us that $\check{R}_{1}$ is full-rank. Then by the hypothesis that Theorem 1.1 has already been proven for domain size $r$, there exists a $2^{\left\lfloor\log _{2} r\right\rfloor} \times r$ basis $M_{(r)}^{\prime}$ on which $\check{R}_{1}, \ldots, \check{R}_{a}$ and $\check{G}_{1}, \ldots, \check{G}_{b}$ are simultaneously realizable. By Theorem $6.7, R_{1}, \ldots, R_{a}$ and $G_{1}, \ldots, G_{b}$ are simultaneously realizable on $2^{\left\lfloor\log _{2} r\right\rfloor} \times k$ basis $M^{\prime}:=$ $M_{(r)}^{\prime} X_{i_{1} \cdots i_{r}}$.

By Corollary 4.4 and Theorem 6.1, it remains to prove collapse theorems for holographic algorithms on domain sizes $k=2^{K}$ and over bases of full rank, after which we get the following corollary.

Corollary 6.8. Any holographic algorithm on a basis of size $\ell$ and domain size $k$ not a power of 2 which uses at least one generator signature of full rank can be simulated on a basis of size at most $2^{\left\lfloor\log _{2} k\right\rfloor}$.

\section{COLLAPSE THEOREM FOR DOMAIN SIZE $2^{K}$}

The following is a direct generalization of the argument from Section 5.3 of [5], but we include it for completeness. We will take $G$ to be a generator signature of full rank on domain size $k=2^{K}$, basis $M$ to be a $2^{\ell} \times 2^{K}$ matrix of rank $2^{K}$, and $\underline{G}=M^{\otimes n} G$ to be the corresponding standard signature of arity $n \ell$. By Theorem 4.2 applied to the transpose of $\underline{G}(t)$, there exists a cluster $Z=s+\left\{e_{p_{1}}, \ldots, e_{p_{K}}\right\}$ of rows of full rank in $\underline{G}(t)$. Denote by $M^{Z}$ the submatrix of $M$ consisting of rows with indices in $Z$. 
LEMMA 7.1. $M^{Z}$ is invertible.

Proof. The $(k, n \ell)$ cluster submatrix of $\underline{G}(t)$ of full rank whose existence is guaranteed by Theorem 4.2 is a submatrix of $M^{Z} G(t)\left(M^{T}\right)^{\otimes(n-1)}$, so $M^{Z}$ has rank at least $2^{K}$. But $M^{Z}$ is a $2^{K} \times 2^{K}$ matrix, so $M^{Z}$ is invertible.

Following the notation of [5], now denote the column vector $\left(M^{Z}\right)^{\otimes n} G$ of dimension $2^{K n}$ by $\underline{G}^{* \leftarrow Z}$, and denote the column vector $\left(M^{Z}\right)^{\otimes(t-1)} \otimes M \otimes\left(M^{Z}\right)^{\otimes(n-t)} \cdot G$ of dimension $2^{K n+\ell-K}$ by $\underline{G}^{t^{c} \leftarrow Z}$. Because $M^{Z}$ and $G(t)$ both have rank $2^{K}, \underline{G}^{* \leftarrow Z}$ and $\underline{G}^{t^{c} \leftarrow Z}$ also have rank $2^{K}$. We check that these can be realized as standard signatures.

LEMMA 7.2. $\underline{G}^{* \leftarrow Z}$ is the standard signature of a generator matchgate of arity $K n$.

Proof. Take the matchgate $G$, and in each block, attach an edge of weight 1 to external node $i(1 \leq i \leq \ell)$ if $s_{i}=1$. In the matchgate $G^{\prime}$ we get from these operations, designate external nodes $p_{1}, \ldots, p_{K}$ in each block as the new external nodes of $G^{\prime}$. The resulting matchgate realizes $\underline{G}^{* \leftarrow Z}$.

LEMMA 7.3. $\underline{G}^{t^{c} \leftarrow Z}$ is the standard signature of a generator matchgate of arity $K n-K+\ell$.

Proof. The proof of Lemma 7.3 is almost identical to that of Lemma 7.2, except block $t$ is treated differently. Take the matchgate $G$, and in each block except the $t$-th one, attach an edge of weight 1 to external node $i(1 \leq i \leq \ell)$ if $s_{i}=1$. In the matchgate $G^{\prime}$ we get from these operations, take the external nodes to be all $\ell$ external nodes in block $t$, as well as nodes $p_{1}, \ldots, p_{K}$ in every other block. The resulting matchgate realizes $\underline{G}^{t^{c} \leftarrow Z}$.

Now define $T=M\left(M^{Z}\right)^{-1}$. Here is the key step of the collapse theorem, making use of Theorem 5.1.

LEMмA 7.4. $T$ is the standard signature of a $K$-input, $\ell-$ output transducer.

Proof. We first express $T$ in terms of $\underline{G}^{* \leftarrow Z}$ and $\underline{G}^{t^{c} \leftarrow Z}$. If the entries of $\underline{G}^{t^{c} \leftarrow Z}$ are indexed by

$$
\begin{aligned}
&\left(i_{1,1} \cdots i_{1, K}\right) \cdots\left(i_{t-1,1} \cdots i_{t-1, K}\right)\left(i_{1}^{\prime} \cdots i_{\ell}^{\prime}\right) \\
&\left(i_{t+1,1} \cdots i_{t+1, \ell}\right) \cdots\left(i_{n, 1} \cdots i_{n, K}\right),
\end{aligned}
$$

denote by $\underline{G}^{t^{c} \leftarrow Z}(t)$ the matrix form of $\underline{G}^{t^{c}} \leftarrow Z$ in which the rows are indexed by $i_{1}^{\prime} \cdots i_{\ell}^{\prime}$ and the columns are indexed by

$$
\begin{aligned}
\left(i_{1,1} \cdots i_{1, K}\right) \cdots( & \left(i_{t-1,1} \cdots i_{t-1, K}\right) \\
& \left(i_{t+1,1} \cdots i_{t+1, \ell}\right) \cdots\left(i_{n, 1} \cdots i_{n, K}\right) .
\end{aligned}
$$

Observe that

$$
\underline{G}=M^{\otimes n} G=T^{\otimes n}\left(M^{Z}\right)^{\otimes n} G=T^{\otimes n} \underline{G}^{* \leftarrow Z}
$$

so that

$$
\underline{G}^{t^{c} \leftarrow Z}=\left(T^{Z}\right)^{\otimes(t-1)} \otimes T \otimes\left(T^{Z}\right)^{\otimes(n-t)} \underline{G}^{* \leftarrow Z} .
$$

Putting both sides of (23) in matrix form, we conclude that

$$
\underline{G}^{t^{c} \leftarrow Z}(t)=T \underline{G}^{* \leftarrow Z}(t) .
$$

Applying Theorem 5.1 to the arity- $K n$ standard signature $\underline{G}^{* \leftarrow Z}$, we have a recognizer whose standard signature $\underline{R}$ satisfies $\underline{G}^{* \leftarrow Z}(t) \underline{R}(t)=I_{2}$. Right-multiplying both sides of (24) by $\underline{R}(t)$, we find that

$$
\underline{G}^{t^{c} \leftarrow Z}(t) \underline{R}(t)=T .
$$

Say that the generator realizing $\underline{G}^{t^{c} \leftarrow Z}$ as a standard signature has external nodes $X_{i, 1}, X_{i, 2}, \ldots, X_{i, K}$ in block $i$ for each $i \neq t$, and external nodes $Y_{t, 1}, \ldots, Y_{t, \ell}$ in block $t$. Say that the generator realizing $\underline{R}$ as a standard signature has external nodes $Z_{i, 1}, \ldots, Z_{i, K}$ in each block $i$.

Construct the transducer $\Gamma$ realizing $T$ as a standard signature by connecting $X_{i, j}$ with $Z_{i, j}$ for all $i \neq t, j \in[K]$. Designate $Y_{t, 1}, \ldots, Y_{t, \ell}$ to be the output nodes of $\Gamma$ and $Z_{t, 1}$, $\ldots, Z_{t, K}$ to be the input nodes of $\Gamma$.

From Theorem 7.4 we obtain the collapse theorem for domain size $2^{K}$.

THEOREM 7.5. Any holographic algorithm on a basis of size $\ell$ and domain size $2^{K}$ which uses at least one generator signature of full rank can be simulated on a basis of size $K$.

Proof. Suppose the holographic algorithm in question uses signatures $R_{i}, G_{j}(1 \leq i \leq r, 1 \leq j \leq g)$ defined by $\underline{R}_{i} M^{\otimes m_{i}}=R_{i}$ and $\underline{G}_{j}=M^{\otimes n_{j}} G_{j}$ over basis $M$. Say that $G_{1}$ has full rank, and let $Z=s+\left\{e_{p_{1}}, \ldots, e_{p_{K}}\right\}$ denote the full-rank $(K, \ell)$-cluster of rows in $G_{1}$ which must exist by Theorem 4.3. By Lemma 7.4, $T:=M\left(M^{Z}\right)^{-1}$ is the standard signature of some transducer matchgate $\Gamma$. Let $\underline{R}_{i}^{\prime}=\underline{R}_{i} T^{\otimes m_{i}}$ and $\underline{G}_{j}^{\prime}=\underline{G}_{j}^{* \leftarrow Z}$; by Lemma $2.4, \underline{R}_{i}^{\prime}$ is the standard signature of some recognizer, and by Lemma 7.3, $\underline{G}_{j}^{\prime}$ is the standard signature of some generator. We conclude that the $R_{i}, G_{j}$ can be simultaneously realized on the basis $M^{Z}$ of size $K$.

\section{ACKNOWLEDGMENTS}

I would like to thank Prof. Leslie Valiant for patiently advising me throughout the course of this project and providing much valuable feedback and suggestions on papers to read. I am extremely indebted to Prof. Jin-Yi Cai for his incredibly extensive and important comments on drafts of this paper. This work was supported in part by NSF \#1509178.

\section{REFERENCES}

[1] J.-Y. Cai and V. Choudhary. Some results on matchgates and holographic algorithms, Automata, languages, and programming. Part 1, Lecture Notes in Comput. Sci., vol 4051, Springer, Berlin, 2006, pp. 703-714.

[2] J.-Y. Cai and V. Choudhary. Valiant's holant theorem and matchgate tensors, Theory and applications of models of computation, Lecture Notes in Comput. Sci., vol. 3959, Springer, Berlin, 2006, pp. 248-261.

[3] J.-Y. Cai and V. Choudhary. Valiant's holant theorem and matchgate tensors, Theoret. Comput. Sci. 384 (2007), no. 1, 22-32.

[4] J.-Y. Cai, V. Choudhary, and P. Lu. On the theory of matchgate computations, Theory Comput. Syst., 45(1):108-132, 2009. Preliminary version in CCC'07.

[5] J.-Y. Cai and Z. Fu. (2014). A collapse theorem for holographic algorithms with matchgates on domain size at most 4. Information and Computation, 239, 149-169.

[6] J.-Y. Cai and A. Gorenstein. Matchgates revisited. CoRR, abs/1303.6729, 2013.

[7] J.-Y. Cai and P. Lu. Holographic algorithms: from art to science, STOC '07-Proceedings of the 39th Annual ACM Symposium on Theory of Computing, AMC, New York, 2007, pp. 401-410. 
[8] J.-Y. Cai and P. Lu: Holographic algorithms: the power of dimensionality resolved. In: Arge, L., Cachin, C., Jurdzinski, T. et al. (eds.) Proceedings of ICALP. Lecture Notes in Computer Science, vol. 4596, pp. 631-642. Springer, Berlin (2007)

[9] J.-Y. Cai and P. Lu. On symmetric signatures in holographic algorithms, STACS 2007, Lecture Notes in Comput. Sci., vol. 4393, Springer, Berlin, 2007, pp. 429-440.

[10] J.-Y. Cai and P. Lu. Basis collapse in holographic algorithms, Computational Complexity 17 (2008), no. 2, 254-281.

[11] Z. Fu, F. Yang: Holographic algorithms on bases of rank 2. http://arxiv/abs/1303.7361. In submission.

[12] P.W. Kasteleyn: The statistics of dimers on a lattice. Physica 27, 1209-1225 (1961)

[13] A. Li and M. Xia: A theory for Valiant's matchcircuits (extended abstract). In Proc. 25th Symp. Theoretical Aspects of Comp. Sci. (STACS'08), pp. 491-502. Schloss Dagstuhl, 2008.

[14] H.N.V. Temperley and M.E. Fisher. Dimer problem in statistical mechanics-an exact result. Philos. Mag. 6, 1061-1063 (1961)

[15] L.G. Valiant. Expressiveness of matchgates, Theoret. Comput Sci. 289 (2002), no. 1, 457-471.

[16] L.G. Valiant. Quantum circuits that can be simulated classically in polynomial time. SIAM J. Comput., 31(4):1229-1254, 2002. Preliminary version in STOC'01.

[17] L.G. Valiant. Accidental algorithms. In FOCS, pages 509-517. IEEE Computer Society, 2006.

[18] L.G. Valiant. Holographic algorithms (extended abstract). In Proc. 45th IEEE Symposium on Foundations of Computer Science, pp. 306-315 (2004).

[19] L.G. Valiant. Holographic circuits, Automata, languages and programming, Lecture Notes in Comput. Sci., vol. 3580, Springer, Berlin, 2005, pp. 1-15.

[20] L.G. Valiant. Some observations on holographic algorithms, Proc. 9th Latin American Theoretical Informatics Symposium, LATIN 2010. LNCS, Vol 6034 Springer-Verlag (2010), 577-590.

\section{APPENDIX}

\section{A. PLANARIZING MATCHGATES}

In the proof of Lemma 5.3, we made several initial constructions of transducers to achieve certain row and column operations but noted that those constructions, shown in Figures 2b, 2c, 2d, needed to be modified because they were not planar. Following the technique of Cai and Gorenstein [6], we planarize those matchgates by replacing every edge crossing with the crossover gadget $X$ shown in Figure 3.

Because the standard signature $X$ of the crossover gadget is given by $\underline{X}^{0000}=1, \underline{X}^{0101}=1, \underline{X}^{1010}=1, \underline{X}^{1111}=-1$, and $\underline{X}^{\sigma}=0$ for all other $\sigma \in\{0,1\}^{4}$ so that the standard signature remains invariant under any cyclic permutation of the external nodes, the orientation of the copy of $X$ placed over an edge crossing does not matter.

We first make precise our operation of planarizing matchgates, following the terminology of [6]. If an edge $\{u, v\}$ of weight $w$ crosses $t$ other edges, replace each of the $t$ crossings by a crossover gadget and replace the edge by $t+1$

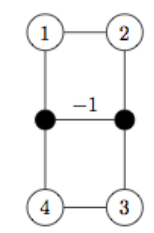

Figure 3: Crossover gadget. Unlabeled edges are of weight 1; external nodes labeled. Figure from [6].

edges connecting adjacent crossover gadgets. Of these $t+1$ edges, assign $t$ of them to have weight 1 and the remaining one to have weight $w$. Call the union of the $t+1$ edges the $u$-v-passage. Given a non-planar matchgate $\Gamma$, denote the matchgate obtained from planarizing $\Gamma$ by $\Gamma^{\prime}$.

OBSERVATION 5. Let $M$ be a perfect matching of $\Gamma^{\prime}$ whose contribution $c$ to PerfMatch $\left(\Gamma^{\prime}\right)$ is nonzero, and let $M^{\prime} \subset M$ denote the edges not belonging to crossover gadgets. Then $M^{\prime}$ is the union of $u$-v-passages corresponds to a perfect matching of $\Gamma$ whose contribution to PerfMatch $(\Gamma)$ is $\pm c$.

PRoof. If an edge incident to one of the external nodes, say node 1 , of a crossover gadget is present in $M$, then the edge incident to node 3 of the crossover gadget must be present in $M$ as well, as $\underline{X}^{\sigma}=0$ if $\sigma_{1} \neq \sigma_{3}$. We conclude that $M$ is a union of $u$-v-passages. The corresponding perfect matching of $\Gamma$ has contribution $\pm c$ because each of the nonzero entries of $\underline{X}$ is \pm 1 .

We need to check that $\underline{\Gamma}$ and $\underline{\Gamma}^{\prime}$ are equal except for a select number of entries differing by a factor of -1 .

Lemma A.1. Let $\Gamma$ be the $K$-input, $K$-output transducer shown in Figure $2 b$ with signature $L_{3}^{j, k}$. There exists a planar matchgate whose standard signature agrees with $\underline{\Gamma}$ on the main diagonal entries and entry $\left(1^{K} \oplus e_{j} \oplus e_{k}, 1^{K}\right)$, and agrees with $\underline{\Gamma}$ everywhere else up to sign.

Proof. Take the desired matchgate to be $\Gamma^{\prime}$. Note that every subgraph of $\Gamma$ has at most one perfect matching. In other words, each entry of $\underline{\Gamma}$ arises from at most a single perfect matching. Therefore, by Observation $5, \underline{\Gamma}$ and $\underline{\Gamma^{\prime}}$ agree everywhere up to sign. Now consider any main diagonal entry $\underline{\Gamma}_{\sigma}^{\prime \sigma}=\operatorname{PerfMatch}\left(\Gamma^{\prime} \backslash Z\right)$. If $M$ is a perfect matching of $\Gamma^{\prime} \backslash \bar{Z}$ making a nonzero contribution to PerfMatch $\left(\Gamma^{\prime} \backslash Z\right)$, it corresponds to a perfect matching of $\Gamma \backslash Z$ making a nonzero contribution to PerfMatch $(\Gamma \backslash Z)$. But the only such perfect matching does not contain the edge between left node $j$ to and left node $k$. Thus, the contribution of this matching and that of $M$ are both equal to 1 .

If $\underline{\Gamma}$ and $\underline{\Gamma^{\prime}}$ disagree on entry $\left(1^{K} \oplus e_{j} \oplus e_{k}, 1^{K}\right)$, modify $\Gamma$ by multiplying the weight of the edge connecting left nodes $j$ and $k$ by -1 , and take the desired matchgate to be the corresponding $\Gamma^{\prime}$.

The following can be proved analogously.

Lemma A.2. Let $\Gamma$ be the transducer in Figure $2 c$ with signature $L_{4}$. There is a planar matchgate whose standard signature agrees with $\underline{\Gamma}$ everywhere up to sign.

LemmA A.3. Let $\Gamma$ be the transducer in Figure 2d with signature $L_{5}^{j}$. There is a planar matchgate whose standard signature agrees with $\underline{\Gamma}$ on the main diagonal entries and entry $\left(1^{K} \oplus e_{j}, 1^{K} \oplus e_{q_{i+1}}\right)$, and everywhere else up to sign. 